\title{
Extensions of almost faithful prime ideals in virtually nilpotent mod- $p$ Iwasawa algebras
}

\author{
William Woods
}

November 5, 2018

\begin{abstract}
Let $G$ be a nilpotent-by-finite compact $p$-adic analytic group for some $p>2$, and $H=\mathbf{F N}_{p}(G)$ its finite-by-(nilpotent $p$-valuable) radical. Fix a finite field $k$ of characteristic $p$, and write $k G$ for the completed group ring of $G$ over $k$. We show that almost faithful $G$-stable prime ideals $P$ of $k H$ extend to prime ideals $P k G$ of $k G \rrbracket$
\end{abstract}

2010 Mathematics Subject Classification: 16S34, 16D25, 16S35, 16W60. 


\section{Contents}

$1 p$-valuations and crossed products 6

1.1 Preliminaries on $p$-valuations $\ldots \ldots \ldots \ldots$

1.2 Ordered bases . . . . . . . . . . . . . . . . . . . . 8

1.3 Separating a free abelian quotient . . . . . . . . . . . 10

1.4 Invariance under the action of a crossed product . . . . . . . . 12

2 A graded ring $\quad 16$

2.1 Generalities on ring filtrations . . . . . . . . . . . . 16

2.2 Constructing a suitable valuation . . . . . . . . . . . 18

2.3 Automorphisms trivial on a free abelian quotient . . . . . . . 24

3 Extending prime ideals from $\mathbf{F N}_{p}(G) \quad 2$

3.1 X-inner automorphisms $\ldots \ldots \ldots \ldots \ldots \ldots$

3.2 Properties of $\mathbf{F N}_{p}(G) \ldots \ldots \ldots \ldots \ldots \ldots$

3.3 The extension theorem . . . . . . . . . . . . . . . . . . . . 30 


\section{Introduction}

Let $G$ be a nilpotent-by-finite compact $p$-adic analytic group and $k$ a finite field of characteristic $p$.

Recall the characteristic open subgroup $H=\mathbf{F N}_{p}(G)$, the finite-by-(nilpotent $p$-valuable) radical of $G$, defined in [10. Theorem C]. This plays an important role in the structure of the group $G$ : for instance, see the structure theorem [10, Theorem D].

In this paper, we demonstrate a connection between certain prime ideals of $k H$ and those of $k G$. The main result of this paper is:

Theorem A. Fix some prime $p>2$. Let $G$ be a nilpotent-by-finite compact $p$-adic analytic group, $H=\mathbf{F N}_{p}(G)$, and $k$ a finite field of characteristic $p$. Let $P$ be an almost faithful, $G$-stable prime ideal of $k H$. Then $P k G$ is a prime ideal of $k G$.

The proof (given in Propositions 3.7 and 3.8) comprises several technical elements, which we outline below.

First, let $G$ be a nilpotent-by-finite compact $p$-adic analytic group with finite radical $\Delta^{+}=1$ [10, Definition 1.2], and let $H=\mathbf{F N}_{p}(G)$. Note that $H$ is $p$ valuable [6, III, 2.1.2], and that $G$ acts on the set of $p$-valuations of $H$ as follows: if $\alpha$ is a $p$-valuation on $H$ and $g \in G$, then we may define a new $p$-valuation $g \cdot \alpha$ on $H$ by

$$
g \cdot \alpha(x)=\alpha\left(g^{-1} x g\right) .
$$

(In fact, we do this in a slightly more general case, but the details are identical. See Lemma 1.17 for the setup.)

Recall the definition of an isolated orbital (closed) subgroup $L$ of $H$ from [10, Definition 1.4], and that normal subgroups are automatically orbital. We show in Definition 1.2 that, if $\omega$ is a $p$-valuation on $H$ and $L$ is a closed isolated normal subgroup of $H$, then $\omega$ induces a quotient $p$-valuation $\Omega$ on $H / L$. We also define the $(t, p)$-filtration (actually a $p$-valuation) on a free abelian pro- $p$ group $A$ of finite rank in Definition 1.4 this is a particularly "uniform" $p$-valuation on $A$, analogous to the $p$-adic valuation $v_{p}$ on $\mathbb{Z}_{p}$.

It is now easy to show the following.

Theorem B. With the above notation: let $L$ be a proper closed isolated normal subgroup of $H$ containing the commutator subgroup $[H, H]$. Then there exists a $p$-valuation $\omega$ on $H$ with the following properties:

(i) $\omega$ is $G$-invariant,

(ii) there exists a real number $t>(p-1)^{-1}$ such that $\left.\omega\right|_{L}>t$, and the quotient $p$-valuation induced by $\omega$ on $G / L$ is the $(t, p)$-filtration. 
Continue to take $G$ to be a nilpotent-by-finite compact $p$-adic analytic group with $\Delta^{+}=1$, and $H=\mathbf{F N}_{p}(G)$. Let $p$ be a prime, $k$ a field of characteristic $p$, and $P$ a faithful prime ideal of $k H$. It is shown in [2, Theorem 8.4] that $P=\mathfrak{p} k H$ for some prime ideal $\mathfrak{p}$ of $k Z$, where $Z$ is the centre of $H$; and, furthermore, in [2, proof of Theorem 8.6], that there exist an integer $e$ and a ring filtration $f$ on $k H / P$ such that

$$
\operatorname{gr}_{f}(k H / P) \cong\left(\operatorname{gr}_{v}(k Z / \mathfrak{p})\right)\left[Y_{1}, \ldots, Y_{e}\right]
$$

where $v=\left.f\right|_{k Z / \mathfrak{p}}$ is a valuation, and $\operatorname{gr}_{f}(k Z / \mathfrak{p})$ is a commutative domain. The valuation $f$ is partly constructed using the $p$-valuation $\omega$ on the group $H$.

Our next theorem is an extension of this result. Write $f_{1}$ for the ring filtration constructed above. Suppose now that $P$ is $G$-stable so that we may consider the ring $k G / P k G$, and fix a crossed product decomposition $(k H / P) * F$ of this ring, where $F=G / H$.

Write $Q^{\prime}$ for the classical ring of quotients of $k Z / \mathfrak{p}$. The filtration $f_{1}$ restricts to a valuation on $k Z / \mathfrak{p}$, which extends naturally to a valuation on $Q^{\prime}$, which we will call $v_{1}$. $F$ acts on the set of valuations of $Q^{\prime}$, and $v_{1}$ has some orbit $\left\{v_{1}, \ldots, v_{s}\right\}$.

Write $Q$ for a certain partial ring of quotients of $k H / P$ containing $Q^{\prime}$. We may naturally form $Q * F$ as an overring of $(k H / P) * F$.

Theorem C. In the above notation: there exists a filtration $\hat{f}$ on $Q * F$ such that

(i) $\operatorname{gr}_{\hat{f}}(Q * F) \cong \operatorname{gr}_{\hat{f}}(Q) * F$, where the right-hand side is some crossed product,

(ii) $\operatorname{gr}_{\hat{f}}(Q) \cong \bigoplus_{i=1}^{s} \operatorname{gr}_{f_{i}}(Q)$,

(iii) $\operatorname{gr}_{f_{i}}(Q) \cong\left(\operatorname{gr}_{v_{i}} Q^{\prime}\right)\left[Y_{1}, \ldots, Y_{e}\right]$ for all $1 \leq i \leq s$,

where $s$ and $e$ are determined as in (†), and the action of $F$ in the crossed product of (i) permutes the $s$ summands in the decomposition of (ii) transitively by conjugation.

We combine Theorems B and $\mathrm{C}$ as follows.

Theorem $\mathrm{C}$, of course, only invokes (ID) in the case when $Q \neq Q^{\prime}$, so we suppose that we are in this case, which occurs precisely when $H$ is non-abelian. Take $L$ to be the smallest closed isolated normal subgroup of $H$ containing both the isolated derived subgroup $H^{\prime}$ [10, Theorem B] and the centre $Z$ of $H$. Now $L$ is a proper subgroup by Lemma 3.5. and we will choose $\omega$ for $L$ as in Theorem B. We may arrange it in (I) and Theorem $\mathrm{C}$ so that, for some $l \leq e$, the elements $Y_{1}, \ldots, Y_{l}$ correspond to a $\mathbb{Z}_{p}$-module basis $x_{1}, \ldots, x_{l}$ for $H / L$; and here the value of the filtration $\hat{f}$ can be understood in terms of the $p$-valuation $\omega$. We show that: 
Theorem D. Take an automorphism $\sigma$ of $H$. Suppose that the induced automorphism on $\operatorname{gr}_{\hat{f}}(Q * F)$ fixes each of the valuations $v_{1}, \ldots, v_{s}$ and fixes each of the elements $Y_{1}, \ldots, Y_{l}$. Then the induced automorphism on $H / L$ (which can be seen as a matrix $M_{\sigma} \in G L_{l}\left(\mathbb{Z}_{p}\right)$ ) lies in the first congruence subgroup of $G L_{l}\left(\mathbb{Z}_{p}\right)$, i.e. it takes the form $M_{\sigma} \in 1+p X$ for some $X \in M_{l}\left(\mathbb{Z}_{p}\right)$. In particular, when $p>2, \sigma$ has finite order if and only if it is the identity automorphism.

A special case of Theorem A, in which $\Delta^{+}=1$, is now deduced from Theorem $\mathrm{D}$ via a long but elementary argument about $\mathrm{X}$-inner automorphisms: see Definition 3.1 and Proposition 3.7 for details.

The case when $\Delta^{+} \neq 1$ now follows as a consequence of the "untwisting" results of [11, Theorems B and C], which allow us to understand the prime ideals of $k H$, along with the conjugation action of $G$, in terms of the corresponding information for $k^{\prime}\left[\left[H / \Delta^{+}\right]\right]$(for various finite field extensions $k^{\prime} / k$ ). Now, as $\Delta^{+}\left(G / \Delta^{+}\right)=1$ and $H / \Delta^{+}=\mathbf{F N}_{p}\left(G / \Delta^{+}\right)$, we are back in the previous case. See Proposition 3.8 for details. 


\section{$1 p$-valuations and crossed products}

\subsection{Preliminaries on $p$-valuations}

Definition 1.1. Recall from [6, III, 2.1.2] that a p-valuation on a group $G$ is a function $\omega: G \rightarrow \mathbb{R} \cup\{\infty\}$ satisfying:

- $\omega\left(x y^{-1}\right) \geq \min \{\omega(x), \omega(y)\}$ for all $x, y \in G$

- $\omega([x, y]) \geq \omega(x)+\omega(y)$ for all $x, y \in G$

- $\omega(x)=\infty$ if and only if $x=1$

- $\omega(x)>\frac{1}{p-1}$ for all $x \in G$

- $\omega\left(x^{p}\right)=\omega(x)+1$ for all $x \in G$.

Throughout this paper we will often be considering several $p$-valuations admitted by a group $G$, so to clarify we may refer to $G$ together with a $p$-valuation $\omega$ as the $p$-valued group $(G, \omega)$ (though when the $p$-valuation in question is clear from context, we will simply write $G$ ).

Given a $p$-valuation $\omega$ on a group $G$, we may write

$$
\begin{aligned}
G_{\omega, \lambda} & :=G_{\lambda}:=\omega^{-1}([\lambda, \infty]), \\
G_{\omega, \lambda^{+}}:=G_{\lambda^{+}} & :=\omega^{-1}((\lambda, \infty])
\end{aligned}
$$

and define the graded group

$$
\operatorname{gr}_{\omega} G:=\bigoplus_{\lambda \in \mathbb{R}} G_{\lambda} / G_{\lambda^{+}} .
$$

Then each element $1 \neq x \in G$ has a principal symbol

$$
\operatorname{gr}_{\omega}(x):=x G_{\mu^{+}} \in G_{\mu} / G_{\mu^{+}} \leq \operatorname{gr}_{\omega} G,
$$

where $\mu$ is defined such that $\mu=\omega(x)$.

Remark. Let $(G, \omega)$ be a $p$-valued group, and $N$ an arbitrary subgroup of $G$. Then $\left(N,\left.\omega\right|_{N}\right)$ is $p$-valued. Moreover, if $G$ has finite rank [6] then so does $N$; and if $G$ is complete with respect to $\omega$ and $N$ is a closed subgroup of $G$, then $N$ is complete with respect to $\left.\omega\right|_{N}$.

Definition 1.2. Given an arbitrary complete $p$-valued group $(G, \omega)$ of finite rank, and a closed isolated normal subgroup $K$ (i.e. a closed normal subgroup $K$ such that $G / K$ is torsion-free), we may define the quotient p-valuation $\Omega$ induced by $\omega$ on $G / K$ as follows:

$$
\Omega(g K)=\sup _{k \in K}\{\omega(g k)\} .
$$


This is defined by Lazard, but the definition is spread across several results, so we collate them here for convenience. The definition in the case of filtered modules is [6, I, 2.1.7], and is modified to the case of filtered groups in [6, the remark after II, 1.1.4.1]. The specialisation from filtered groups to $p$-saturable groups is done in [6, III, 3.3.2.4], where it is proved that $\Omega$ is indeed still a $p$-valuation on $G / K$; and the general case is stated in [6. III, 3.1.7.6], and eventually proved in [6, IV, 3.4.2].

As a partial inverse to the above process of passing to a quotient $p$-valuation, we prove the following general result about "lifting" $p$-valuations from torsion-free quotients.

Theorem 1.3. Let $G$ be a complete $p$-valued group of finite rank, and $N$ a closed isolated orbital (hence normal) subgroup of $G$. Suppose we are given two functions

$$
\alpha, \beta: G \rightarrow \mathbb{R} \cup\{\infty\},
$$

such that $\alpha$ is a $p$-valuation on $G$, and $\beta$ factors through a $p$-valuation on $G / N$, i.e.

$$
\bar{\beta}: G / N \rightarrow \mathbb{R} \cup\{\infty\} .
$$

Then $\omega=\inf \{\alpha, \beta\}$ is a $p$-valuation on $G$.

Proof. $\alpha$ and $\beta$ are both filtrations on $G$ (in the sense of [6. II, 1.1.1]), and so by [6, II, 1.2.10], $\omega$ is also a filtration. Following [6, III, 2.1.2], for $\omega$ to be a $p$-valuation, we need to check the following three conditions:

(i) $\omega(x)<\infty$ for all $x \in G, x \neq 1$.

This follows from the fact that $\alpha$ is a $p$-valuation, and hence $\alpha(x)<\infty$ for all $x \in G, x \neq 1$.

(ii) $\omega(x)>(p-1)^{-1}$ for all $x \in G$.

This follows from the fact that $\alpha(x)>(p-1)^{-1}$ and $\beta(x)>(p-1)^{-1}$ for all $x \in G$ by definition.

(iii) $\omega\left(x^{p}\right)=\omega(x)+1$ for all $x \in G$.

Take any $x \in G$. As $\alpha$ is a $p$-valuation, we have by definition that $\alpha\left(x^{p}\right)=\alpha(x)+1$.

If $x \in N$, this alone is enough to establish the condition, as $\left.\omega\right|_{N}=\left.\alpha\right|_{N}$ (since $\beta(x)=\infty$ ).

Suppose instead that $x \in G \backslash N$. Then, as $N$ is assumed isolated orbital in $G$, we also have $x^{p} \in G \backslash N$, so by definition of $\beta$ we have

$$
\beta\left(x^{p}\right)=\bar{\beta}\left((x N)^{p}\right)=\bar{\beta}(x N)+1=\beta(x)+1,
$$

with the middle equality coming from the fact that $\bar{\beta}$ is a $p$-valuation. Now it is clear that $\omega\left(x^{p}\right)=\omega(x)+1$ by definition of $\omega$. 
Finally, the following function will be crucial. (It is in fact a $p$-valuation, but we delay the proof of this fact until Lemma 1.6.)

Definition 1.4. Let $A$ be a free abelian pro- $p$ group of rank $d>0$ (here written multiplicatively). Choose a real number $t>(p-1)^{-1}$. Then the $(t, p)$-filtration on $A$ is the function $\omega: A \rightarrow \mathbb{R} \cup\{\infty\}$ defined by

$$
\omega(x)=t+n,
$$

where $n$ is the non-negative integer such that $x \in A^{p^{n}} \backslash A^{p^{n-1}}$. (By convention, $\omega(1)=\infty$.)

\subsection{Ordered bases}

Definition 1.5. Recall from 2, 4.2] that an ordered basis for a $p$-valued group $(G, \omega)$ is a set $\left\{g_{1}, \ldots, g_{e}\right\}$ of elements of $G$ such that every element $x \in G$ can be uniquely written as the (ordered) product

$$
x=\prod_{1 \leq i \leq e} g_{i}^{\lambda_{i}}
$$

for some $\lambda_{i} \in \mathbb{Z}_{p}$, and

$$
\omega(x)=\inf _{1 \leq i \leq e}\left\{\omega\left(g_{i}\right)+v_{p}\left(\lambda_{i}\right)\right\},
$$

where $v_{p}$ is the usual $p$-adic valuation on $\mathbb{Z}_{p}$. (Note that an ordered basis for $(G, \omega)$ need not be an ordered basis for $\left(G, \omega^{\prime}\right)$ for another $p$-valuation $\omega^{\prime}$.)

As in 2, we will often write

$$
\mathrm{g}^{\lambda}:=\prod_{1 \leq i \leq e} g_{i}^{\lambda_{i}}
$$

as shorthand, where $\lambda \in \mathbb{Z}_{p}^{e}$.

We now show that the function given in Definition 1.4 is indeed a $p$-valuation, and demonstrate some of its properties.

Lemma 1.6. Let $A$ and $t$ be as in Definition 1.4

(i) The $(t, p)$-filtration $\omega$ is a $p$-valuation on $A$.

(ii) Suppose we are given a $\mathbb{Z}_{p}$-module basis $B=\left\{a_{1}, \ldots, a_{d}\right\}$ for $A$, and a $p$-valuation $\alpha$ on $A$ satisfying $\alpha\left(a_{1}\right)=\cdots=\alpha\left(a_{d}\right)=t$. Then $\alpha$ is the $(t, p)$-filtration on $A$, and $B$ is an ordered basis for $(A, \alpha)$.

(iii) The $(t, p)$-filtration $\omega$ is completely invariant under automorphisms of $A$, i.e. the subgroups $A_{\omega, \lambda}$ and $A_{\omega, \lambda^{+}}$are characteristic in $A$.

Proof. 
(i) This is a trivial check from the definition [6, III, 2.1.2].

(ii) By [6, III, 2.2.4], we see that

$$
\alpha\left(a_{1}^{\lambda_{1}} \ldots a_{d}^{\lambda_{d}}\right)=t+\inf _{1 \leq i \leq d}\left\{v_{p}\left(\lambda_{i}\right)\right\},
$$

which is precisely the $(t, p)$-filtration.

(iii) The subgroups $A^{p^{n}}$ are clearly characteristic in $A$.

Remark. The $(t, p)$-filtration as defined here is equivalent to the definition given in [6. II, 3.2.1] for free abelian pro- $p$ groups of finite rank.

Recall from [10, Definitions 1.1 and 1.4] that a closed subgroup $H$ of a profinite group $G$ is $(G$-)orbital if it has finitely many $G$-conjugates, and isolated orbital if any $G$-orbital $H^{\prime} \gtrless H$ satisfies $\left[H^{\prime}: H\right]=\infty$.

The following is a general property of ordered bases.

Lemma 1.7. Let $(G, \omega)$ be a complete $p$-valued group of finite rank, and $N$ a closed isolated normal subgroup of $G$. Then there exist sets $B_{N} \subseteq B_{G}$ such that $B_{N}$ is an ordered basis for $\left(N,\left.\omega\right|_{N}\right)$ and $B_{G}$ is an ordered basis for $(G, \omega)$.

Proof. This was established in [2, proof of Lemma 8.5(a)].

Remark. It may be helpful to think of this as follows:

$$
B_{G}=\{\underbrace{x_{1}, \ldots, x_{r}}_{B_{G / N}}, \underbrace{x_{r+1}, \ldots, x_{s}}_{B_{N}}\},
$$

where $B_{G / N}=B_{G} \backslash B_{N}$ is in fact some appropriate preimage in $G$ of any ordered basis for $(G / N, \Omega)$, where $\Omega$ is the quotient $p$-valuation.

Lemma 1.8. Let $(G, \alpha)$ be a complete $p$-valued group of finite rank, and $N$ a closed isolated orbital (hence normal) subgroup of $G$. Take also a $p$-valuation $\bar{\beta}$ on $G / N$. Suppose we are given sets

$$
B_{G}=\{\underbrace{x_{1}, \ldots, x_{r}}_{B_{G / N}}, \underbrace{x_{r+1}, \ldots, x_{s}}_{B_{N}}\}
$$

such that

- $B_{N}$ is an ordered basis for $\left(N,\left.\alpha\right|_{N}\right)$,

- $B_{G}$ is an ordered basis for $(G, \alpha)$, and

- the image in $G / N$ of $B_{G / N}$ is an ordered basis for $(G / N, \bar{\beta})$.

In the notation of Theorem 1.3, write $\beta$ for the composite of $G \rightarrow G / N$ with $\bar{\beta}$, and form the $p$-valuation $\omega=\inf \{\alpha, \beta\}$ for $G$.

Then $B_{G}$ is an ordered basis for $(G, \omega)$. 
Proof. We need only check that

$$
\omega\left(\mathbf{x}^{\lambda}\right)=\inf _{1 \leq i \leq s}\left\{\omega\left(x_{i}\right)+v_{p}\left(\lambda_{i}\right)\right\}
$$

for any $\lambda \in \mathbb{Z}_{p}^{s}$. But we have by definition that

$$
\begin{aligned}
& \alpha\left(\mathbf{x}^{\lambda}\right)=\inf _{1 \leq i \leq s}\left\{\alpha\left(x_{i}\right)+v_{p}\left(\lambda_{i}\right)\right\}, \\
& \beta\left(\mathbf{x}^{\lambda}\right)=\inf _{1 \leq i \leq r}\left\{\beta\left(x_{i}\right)+v_{p}\left(\lambda_{i}\right)\right\},
\end{aligned}
$$

and the result follows trivially.

\subsection{Separating a free abelian quotient}

Results in later sections will require the existence of a $p$-valuation on an appropriate group $G$ satisfying a certain technical property, which we can now finally state:

Definition 1.9. Let $(G, \omega)$ be a complete $p$-valued group of finite rank, and $L$ a closed isolated normal subgroup containing $[G, G]$ (and hence containing the isolated derived subgroup $G^{\prime}$, which was defined and written as $G^{(1)}$ in 10, Theorem B]). We will say that $\omega$ satisfies property $\left(\mathrm{A}_{L}\right)$ if there is an ordered basis $\left\{g_{d+1}, \ldots, g_{e}\right\}$ for $\left(L,\left.\omega\right|_{L}\right)$, contained in an ordered basis $\left\{g_{1}, \ldots, g_{e}\right\}$ for $(G, \omega)$ (e.g. constructed by Lemma 1.7), such that the following hold:

and, for all $\ell \in L$,

$$
\left.\begin{array}{r}
\omega\left(g_{1}\right)=\cdots=\omega\left(g_{d}\right), \\
\omega\left(g_{1}\right)<\omega(\ell) .
\end{array}\right\}
$$

Remark. In the notation of the above definition, suppose that $\omega$ satisfies $\omega\left(g_{1}\right)<\omega(\ell)$ for all $\ell \in L$. Then, by our earlier remarks, we note that the condition $\left(\mathrm{A}_{L}\right)$ is equivalent to the statement that the quotient $p$-valuation induced by $\omega$ on $G / L$ is the $(t, p)$-filtration for $t:=\omega\left(g_{1}\right)$.

Definition 1.10. Following [6, III, 2.1.2], we will say that a group $G$ is $p$ valuable if there exists a $p$-valuation $\omega$ for $G$, and $G$ is complete with respect to $\omega$ and has finite rank.

Lemma 1.11. Let $G$ be a nilpotent $p$-valuable group, and $L$ a closed isolated normal subgroup containing $G^{\prime}$. Then there exists some $p$-valuation $\omega$ for $G$ satisfying $\left(A_{L}\right)$.

Proof. Let $\alpha$ be a $p$-valuation on $G$. Take an ordered basis $\left\{g_{d+1}, \ldots, g_{e}\right\}$ for $\left(L,\left.\alpha\right|_{L}\right)$ and extend it to an ordered basis $\left\{g_{1}, \ldots, g_{e}\right\}$ for $(G, \alpha)$ by Lemma 1.7 Fix a number $t$ satisfying

$$
(p-1)^{-1}<t \leq \inf _{1 \leq i \leq e} \alpha\left(g_{i}\right) .
$$


Applying Theorem 1.3 with $N=L$ and $\bar{\beta}$ the $(t, p)$-filtration on $G / L$, we see that $\omega=\inf \{\alpha, \beta\}$ is a $p$-valuation for $G$; and by Lemma 1.8, $\left\{g_{1}, \ldots, g_{e}\right\}$ is still an ordered basis for $(G, \omega)$, so we can check easily that $\omega$ satisfies $\left(\mathrm{A}_{L}\right)$ by construction.

Remark. In fact, by analysing the construction in Theorem 1.3, we can see that we have shown something stronger: that any $p$-valuation $\alpha$ may be refined to such an $\omega$ satisfying $\left.\omega\right|_{L}=\left.\alpha\right|_{L}$. But we will not use this fact in this paper.

Remark. If $\omega$ satisfies $\left(\mathrm{A}_{L}\right)$ as above, write $t:=\omega\left(g_{1}\right)$. Then, for any automorphism $\sigma$ of $G$ and any $1 \leq i \leq d$, we have

$$
\omega\left(\sigma\left(g_{i}\right)\right)=t
$$

This follows from Lemma 1.6(iii). Indeed, by construction, we have $G_{t}=G$, and $G_{t^{+}}=G^{p} \cdot L$, an open normal subgroup; and when $L$ is characteristic, $G_{t^{+}}$ is characteristic.

Now let $G$ be a $p$-valuable group with fixed $p$-valuation $\omega$, and let $\sigma \in \operatorname{Aut}(G)$. In this subsection and the next, we seek to establish conditions under which a given automorphism $\sigma$ of $G$ will preserve the "dominant" part of certain elements $x \in G$ (with respect to $\omega$ ). That is, we are looking for a condition under which

$$
\operatorname{gr}_{\omega}(\sigma(x))=\operatorname{gr}_{\omega}(x) .
$$

Clearly it is necessary and sufficient that the following holds:

$$
\omega\left(\sigma(x) x^{-1}\right)>\omega(x) .
$$

The results of this paper rely on our ability to invoke the following technical result.

Theorem 1.12. Let $G$ be a $p$-valuable group, and let $L$ be a proper closed isolated orbital (hence normal) subgroup containing $[G, G]$, so that we have an isomorphism $\varphi: G / L \rightarrow \mathbb{Z}_{p}^{d}$ for some $d \geq 1$. Write $q: G \rightarrow G / L$ for the natural quotient map.

Choose a $\mathbb{Z}_{p}$-basis $\left\{e_{1}, \ldots, e_{d}\right\}$ for $\mathbb{Z}_{p}^{d}$. For each $1 \leq i \leq d$, fix an element $g_{i} \in G$ with $\varphi \circ q\left(g_{i}\right)=e_{i}$. Fix an automorphism $\sigma$ of $G$ preserving $L$, so that $\sigma$ induces an automorphism $\bar{\sigma}$ of $G / L$, and hence an automorphism $\hat{\sigma}=\varphi \circ \bar{\sigma} \circ \varphi^{-1}$ of $\mathbb{Z}_{p}^{d}$. Let $M_{\sigma}$ be the matrix of $\hat{\sigma}$ with respect to the basis $\left\{e_{1}, \ldots, e_{d}\right\}$.

Suppose there exists some $p$-valuation $\omega$ on $G$ with the following properties:

(i) (1.1) holds for all $x \in\left\{g_{1}, \ldots, g_{d}\right\}$,

(ii) $\omega\left(g_{1}\right)=\cdots=\omega\left(g_{d}\right)(=t$, say),

(iii) $\omega(\ell)>t$ for all $\ell \in L$.

Then $M_{\sigma}-1 \in p M_{d}\left(\mathbb{Z}_{p}\right)$. 
Remark. Conditions (ii) and (iii) are just the statement that $\omega$ satisfies $\left(A_{L}\right)$.

Proof. Define the function $\Omega: \mathbb{Z}_{p}^{d} \rightarrow \mathbb{R} \cup\{\infty\}$ by

$$
\Omega \circ \varphi(g L)=\sup _{\ell \in L}\{\omega(g \ell)\} .
$$

By the remarks made in Definition $1.2 \Omega$ is in fact a $p$-valuation.

By assumption (iii), we see that, for each $1 \leq i \leq d$ and any $\ell \in L$, we have $\omega\left(g_{i}\right)=\omega\left(g_{i} \ell\right)$, so that

$$
\Omega\left(e_{i}\right)=\Omega \circ \varphi\left(g_{i} L\right)=\sup _{\ell \in L}\left\{\omega\left(g_{i} \ell\right)\right\}=\omega\left(g_{i}\right),
$$

so by assumption (ii), $\Omega\left(e_{i}\right)=t$. Hence, by Lemma 1.6(ii), $\Omega$ must be the $(t, p)$-filtration on $\mathbb{Z}_{p}^{d}$. Now, by assumption (i), we have

$$
\Omega(\hat{\sigma}(x)-x)>t
$$

for all $x \in\left\{e_{1}, \ldots, e_{d}\right\}$, and hence, as $\Omega-t$ takes integer values (by Definition 1.4),

$$
\Omega(\hat{\sigma}(x)-x) \geq t+1
$$

and so $\hat{\sigma}(x)-x \in p \mathbb{Z}_{p}^{d}$ for each $x \in\left\{e_{1}, \ldots, e_{d}\right\}$, which is what we wanted to prove.

Remark. With Lemma 1.6 in mind, we note the following: suppose $\omega$ satisfies hypothesis (iii) of Theorem 1.12. Then hypothesis (ii) is equivalent to the statement that the quotient filtration induced by $\omega$ on $G / L$ is actually the $(t, p)$-filtration on $G / L$.

\subsection{Invariance under the action of a crossed product}

Definition 1.13. Let $R$ be a ring, and fix a subgroup $G \leq R^{\times}$; let $F$ be a group. Fix a crossed product

$$
S=R \underset{\langle\sigma, \tau\rangle}{*} F .
$$

Consider the following properties that this crossed product may satisfy:

The image $\sigma(F)$ normalises $G$, i.e. $x^{\sigma(f)} \in G$ for all $x \in G, f \in F . \quad\left(\mathrm{N}_{G}\right)$

The image $\tau(F, F)$ normalises $G$. $\quad\left(\mathrm{N}_{G}^{\prime}\right)$

The image $\tau(F, F)$ is a subset of $G$. $\quad\left(\mathrm{P}_{G}\right)$

In the case when $G$ is $p$-valuable, consider the set of $p$-valuations of $G$. Then $\operatorname{Aut}(G)$ acts on this set as follows:

$$
(\varphi \cdot \omega)(x)=\omega\left(x^{\varphi}\right)
$$


When $S$ satisfies $\left(\mathrm{N}_{G}^{\prime}\right), \tau(F, F) \subseteq G$, so we get a map $\rho: \tau(F, F) \rightarrow \operatorname{Inn}(G)$ (with elements of $G$ acting by conjugation), so we will also consider the following property:

Every $p$-valuation $\omega$ of $G$ is invariant under elements of $\tau(F, F) . \quad\left(\mathrm{Q}_{G}\right)$

Lemma 1.14. In the notation above:

(i) If $S$ satisfies $\left(\mathrm{N}_{G}\right)$, then $S$ satisfies $\left(\mathrm{N}_{G}^{\prime}\right)$.

(ii) If $S$ satisfies $\left(\mathrm{P}_{G}\right)$, then $S$ satisfies $\left(\mathrm{N}_{G}^{\prime}\right)$.

(iii) If $S$ satisfies $\left(\mathrm{P}_{G}\right)$, then $S$ satisfies $\left(\mathrm{Q}_{G}\right)$.

Proof.

(i) Note that $\rho \circ \tau(x, y)=\sigma(x y)^{-1} \sigma(x) \sigma(y)$.

(ii) Obvious.

(iii) By (ii), we see that $S$ satisfies $\left(\mathrm{N}_{G}^{\prime}\right)$, so it makes sense to consider $\left(\mathrm{Q}_{G}\right)$.

Let $\omega$ be a $p$-valuation of $G$, and take $t \in \tau(F, F)$. As $S$ satisfies $\left(\mathrm{P}_{G}\right)$, we actually have $t \in G$. Then, for any $x \in G$, we have

$$
\begin{aligned}
(t \cdot \omega)(x) & =\omega\left(x^{t}\right) \\
& =\omega\left(t^{-1} x t\right) \\
& =\omega(x \cdot[x, t]) \\
& \geq \min \{\omega(x), \omega([x, t])\}=\omega(x),
\end{aligned}
$$

and so (by symmetry) $\omega\left(t^{-1} x t\right)=\omega^{\prime}(x)$.

Definition 1.15. Recall, from [11, Definition 5.4], that if we have a fixed crossed product

$$
S=R \underset{\langle\sigma, \tau\rangle}{*} F
$$

and a 2-cocycle

$$
\alpha \in Z_{\sigma}^{2}\left(F, Z\left(R^{\times}\right)\right)
$$

then we may define the ring

$$
S_{\alpha}=R \underset{\langle\sigma, \tau \alpha\rangle}{*} F
$$

the 2-cocycle twist (of $R$, by $\alpha$, with respect to the decomposition (1.2)).

Lemma 1.16. Continuing with the notation above,

(i) $S$ satisfies $\left(\mathrm{N}_{G}\right)$ if and only if $S_{\alpha}$ satisfies $\left(\mathrm{N}_{G}\right)$. 
(ii) $S$ satisfies $\left(\mathrm{Q}_{G}\right)$ if and only if $S_{\alpha}$ satisfies $\left(\mathrm{Q}_{G}\right)$.

Proof.

(i) Trivial from Definitions 1.13 and 1.15 .

(ii) As $\alpha(F, F) \subseteq Z(R)^{\times}$, conjugation by $\alpha$ is the identity automorphism on $G$.

These properties will be interesting to us later as they will allow us to invoke the following lemma:

Lemma 1.17. If $S$ satisfies $\left(\mathrm{N}_{G}\right)$, then, given any $g \in F$ and $p$-valuation $\omega$ on $G$, the function $g \cdot \omega$ given by

$$
(g \cdot \omega)(x)=\omega\left(x^{\sigma(g)}\right)
$$

is again a $p$-valuation on $G$. If, further, $S$ satisfies $\left(\mathrm{Q}_{G}\right)$, then this is a group action of $F$ on the set of $p$-valuations of $G$.

Proof. If $x \in G$, then $x^{\sigma(g)} \in G$ because $S$ satisfies ( $\mathrm{N}_{G}$ ), so it makes sense to consider $\omega\left(x^{\sigma(g)}\right)$. The definition above does indeed give a group action when $S$ satisfies $\left(\mathrm{Q}_{G}\right)$, as, for all $g, h \in F$,

$$
\begin{array}{rlr}
(g \cdot(h \cdot \omega))(x) & =h \cdot \omega\left(x^{\sigma(g)}\right) & \\
& =\omega\left(x^{\sigma(g) \sigma(h)}\right) & \\
& =\omega\left(x^{\sigma(g h) \tau(g, h)}\right) & \\
& =\omega\left(x^{\sigma(g h)}\right) & \text { by }\left(Q_{G}\right) \\
& =(g h \cdot \omega)(x) . &
\end{array}
$$

The following lemma will allow us to prove the existence of a sufficiently "nice" $p$-valuation.

Lemma 1.18. Suppose $S$ satisfies $\left(\mathrm{N}_{G}\right)$ and $\left(\mathrm{Q}_{G}\right)$, so that $\sigma$ induces an action of $F$ on the set of $p$-valuations on $G$ as in the above lemma. Let $\omega$ be a $p$ valuation on $G$. If the $F$-orbit of $\omega$ is finite, then $\omega^{\prime}(x)=\inf _{g \in F}(g \cdot \omega)(x)$ defines an $F$-invariant $p$-valuation on $G$.

Furthermore, if $L$ is a closed isolated characteristic subgroup of $G$ containing $G^{\prime}$, and $\omega$ satisfies $\left(\mathrm{A}_{L}\right)$ (as in Definition 1.9), then $\omega^{\prime}$ satisfies $\left(\mathrm{A}_{L}\right)$.

Proof. The function $\omega^{\prime}$ satisfies condition [6, III, 2.1.2.2], since the F-orbit of $\omega$ is finite, and is hence a $p$-valuation that is $F$-stable by the remark in [6, III, 2.1.2].

Suppose $\omega$ satisfies $\left(\mathrm{A}_{L}\right)$. That is, for some $t>(p-1)^{-1}, \omega$ induces the $(t, p)$-filtration on $G / L$, and $\omega(\ell)>t$ for all $\ell \in L$. But, given any $g \in F$, 
clearly $g \cdot \omega$ still induces the $(t, p)$-filtration on $G / L$ by Lemma 1.6(iii), and $(g \cdot \omega)(\ell)=\omega\left(\ell^{\sigma(g)}\right)>t$, since $\ell^{\sigma(g)} \in L$ as $L$ is characteristic. Taking the infimum over the finitely many distinct $g \cdot \omega, g \in F$, shows that $\omega^{\prime}$ also satisfies $\left(A_{L}\right)$.

Recall the finite radical $\Delta^{+}=\Delta^{+}(G)$ from [10, Definition 1.2].

Definition 1.19. Let $G$ be an arbitrary compact $p$-adic analytic group with $\Delta^{+}=1, H$ an open normal subgroup of $G, F=G / H$, and $P$ a faithful $G$ stable ideal of $k H$. Recall from [11, Definition 5.11] that the crossed product decomposition

$$
k G / P k G=k H / P \underset{\langle\sigma, \tau\rangle}{*} F
$$

is standard if the basis $\bar{F}$ is a subset of the image of the map $G \hookrightarrow(k G / P k G)^{\times}$.

Lemma 1.20. Suppose that $k G / P k G=k H / P \underset{\langle\sigma, \tau\rangle}{*} F$ is a standard crossed product decomposition. Take any $\alpha \in Z_{\sigma}^{2}\left(F, Z\left((k H / P)^{\times}\right)\right)$, and form the central 2-cocycle twist

$$
(k G / P k G)_{\alpha}:=k H / P \underset{\langle\sigma, \tau \alpha\rangle}{*} F
$$

with respect to this decomposition [11, Definition 5.4].

Consider $H$ as a subgroup of $(k H / P)^{\times}$: then conjugation by elements of $\bar{G}$ inside $\left((k G / P k G)_{\alpha}\right)^{\times}$induces a group action of $F$ on the set of $p$-valuations of $H$, as in Lemma 1.17 .

Remark. As the crossed product notation suggests, this lemma simply says that the action of $F$ on $H$, via $\sigma$, is unchanged after applying $(-)_{\alpha}$.

Proof. As the decomposition is standard, $k G / P k G$ trivially satisfies both $\left(\mathrm{N}_{H}\right)$ (as $H$ is normal in $G$ ) and $\left(\mathrm{P}_{H}\right)$. By Lemma 1.14(iii), $k G / P k G$ also satisfies $\left(\mathrm{Q}_{H}\right)$. Now Lemma 1.16] shows that $(k G / P k G)_{\alpha}$ also satisfies $\left(\mathrm{N}_{H}\right)$ and $\left(\mathrm{Q}_{H}\right)$, so that $\sigma$ induces a group action of $F$ on the $p$-valuations of $H$ inside $(k G / P k G)_{\alpha}$ by Lemma 1.17 .

Let $L$ be a closed isolated characteristic subgroup of $H$ containing $[H, H]$.

Corollary 1.21. With notation as above, we can find an $F$-stable $p$-valuation $\omega$ on $H$ satisfying $\left(\mathrm{A}_{L}\right)$.

Proof. This now follows immediately from Lemmas 1.11 and 1.18 .

Proof of Theorem B. This follows from Corollary 1.21, 


\section{$2 \quad$ A graded ring}

\subsection{Generalities on ring filtrations}

Definition 2.1. Recall that a filtration $v$ on the ring $R$ is a function $v: R \rightarrow \mathbb{R} \cup\{\infty\}$ satisfying, for all $x, y \in R$,

- $v(x+y) \geq \min \{v(x), v(y)\}$,

- $v(x y) \geq v(x)+v(y)$,

- $v(0)=\infty, v(1)=0$.

If in addition we have $v(x y)=v(x)+v(y)$ for all $x, y \in R$, then $v$ is a valuation on $R$.

First, a basic property of ring filtrations.

Lemma 2.2. Suppose $v$ is a filtration on $R$ which takes non-negative values, i.e. $v(R) \subseteq[0, \infty]$, and let $u \in R^{\times}$. Then $v(u x)=v(x u)=v(x)$ for all $x \in R$.

Proof. By the definition of $v$, we have $0=v(1)=v\left(u u^{-1}\right) \geq v(u)+v\left(u^{-1}\right)$. As $v(u) \geq 0$ and $v\left(u^{-1}\right) \geq 0$, we must have $v(u)=0=v\left(u^{-1}\right)$. Then

$$
v(x)=v\left(u^{-1} u x\right) \geq v\left(u^{-1}\right)+v(u x)=v(u x) \geq v(u)+v(x)=v(x),
$$

from which we see that $v(x)=v(u x)$; and by a symmetric argument, we also have $v(x u)=v(x)$.

We will fix the following notation for this subsection.

Notation. Let $G$ be a $p$-valuable group equipped with the fixed $p$-valuation $\omega$, and $k$ a field of characteristic $p$. Take an ordered basis $\left\{g_{1}, \ldots, g_{d}\right\}$ for $G$, and write $b_{i}=g_{1}-1 \in k G$ for all $1 \leq i \leq d$. As in [2], we make the following definitions:

- for each $\alpha \in \mathbb{N}^{d}, \mathbf{b}^{\alpha}$ means the (ordered) product $b_{1}^{\alpha_{1}} \ldots b_{d}^{\alpha_{d}} \in k G$,

- for each $\alpha \in \mathbb{Z}_{p}^{d}, \mathbf{g}^{\alpha}$ means the (ordered) product $g_{1}^{\alpha_{1}} \ldots g_{d}^{\alpha_{d}} \in G$,

- for each $\alpha \in \mathbb{N}^{d},\langle\alpha, \omega(\mathbf{g})\rangle$ means $\sum_{i=1}^{d} \alpha_{i} \omega\left(g_{i}\right)$,

- the canonical ring homomorphism $\mathbb{Z}_{p} \rightarrow k$ will sometimes be left implicit, but will be denoted by $\iota$ when necessary for clarity.

Definition 2.3. With notation as above, let $w$ be the valuation on $k G$ defined in [2, 6.2], given by

$$
\sum_{\alpha \in \mathbb{N}^{d}} \lambda_{\alpha} \mathbf{b}^{\alpha} \mapsto \inf _{\alpha \in \mathbb{N}^{d}}\left\{\langle\alpha, \omega(\mathbf{g})\rangle \mid \lambda_{\alpha} \neq 0\right\} .
$$


Note that, in light of this formula [2, Corollary 6.2(b)], and by the construction [6. III, 2.3.3] of $w$, it is clear that the value of $w$ is in fact independent of the ordered basis chosen. In particular, if $\varphi$ is an automorphism of $G$, then $\left\{g_{1}^{\varphi}, \ldots, g_{d}^{\varphi}\right\}$ is another ordered basis of $G$; hence if $\omega$ is $\varphi$-stable (in the sense that $\omega\left(g^{\varphi}\right)=\omega(g)$ for all $g \in G$ ), then $w$ is $\varphi$-stable (in the sense that $w\left(x^{\hat{\varphi}}\right)=w(x)$ for all $x \in k G$, where $\hat{\varphi}$ here denotes the natural extension of $\varphi$ to $k G$, obtained by the universal property [11, Lemma 2.2]).

We will need the following result:

Lemma 2.4. Let

$$
\begin{gathered}
b=b_{0}+b_{1} p+b_{2} p^{2}+\cdots \in \mathbb{Z}_{p}, \\
n=n_{0}+n_{1} p+n_{2} p^{2}+\cdots+n_{s} p^{s} \in \mathbb{N},
\end{gathered}
$$

where all $b_{i}, n_{i} \in\{0,1, \ldots, p-1\}$. Then

$$
\left(\begin{array}{l}
b \\
n
\end{array}\right) \equiv \prod_{i=0}^{s}\left(\begin{array}{c}
b_{i} \\
n_{i}
\end{array}\right) \quad \bmod p .
$$

Proof. See e.g. [1, Theorem].

Corollary 2.5. Let $b \in \mathbb{Z}_{p}, n \in \mathbb{N}$. If

$$
v_{p}\left(\left(\begin{array}{l}
b \\
n
\end{array}\right)\right)=0
$$

then $v_{p}(b) \leq v_{p}(n)$. Further, for fixed $b \in \mathbb{Z}_{p}$,

$$
\inf \left\{n \in \mathbb{N} \mid v_{p}\left(\left(\begin{array}{c}
b \\
n
\end{array}\right)\right)=0\right\}=p^{v_{p}(b)} .
$$

Proof. From Lemma 2.4 above, we can see that

$$
\left(\begin{array}{c}
b \\
n
\end{array}\right) \equiv 0 \quad \bmod p
$$

if and only if, for some $0 \leq i \leq s$,

$$
\left(\begin{array}{c}
b_{i} \\
n_{i}
\end{array}\right)=0
$$

which happens if and only if one of the pairs $\left(b_{i}, n_{i}\right)$ for $0 \leq i \leq s$ has $b_{i}=0 \neq n_{i}$. Hence, to ensure that this does not happen, we must have $v_{p}(b) \leq v_{p}(n)$. It is clear from Lemma 2.4 that $n=p^{v_{p}(b)}$ satisfies (2.1), and is the least $n \in \mathbb{N}$ with $v_{p}(b) \leq v_{p}(n)$.

Theorem 2.6. Take any $x \in G$, and $t=\inf \omega(G)$. Then $w(x-1)>t$ implies $\omega(x)>t$. 
Proof. Write $x=\mathbf{g}^{\alpha}$. In order to show that $\omega\left(\mathbf{g}^{\alpha}\right)>t$, it suffices to show that $\omega\left(g_{j}\right)+v_{p}\left(\alpha_{j}\right)>t$ for each $j$ (as there are only finitely many), and hence that $v_{p}\left(\alpha_{j}\right) \geq 1$ for all $j$ such that $\omega\left(g_{j}\right)=t$. This is equivalent to the claim that $p^{v_{p}\left(\alpha_{j}\right)}>1$, which we will write as $p^{v_{p}\left(\alpha_{j}\right)} \omega\left(g_{j}\right)>t$ for all $j$ with $\omega\left(g_{j}\right)=t$.

Let $\beta^{(j)}$ be the $d$-tuple with $i$ th entry $\delta_{i j} p^{v_{p}\left(\alpha_{j}\right)}$. Then, of course,

$$
\left\langle\beta^{(j)}, \omega(\mathbf{g})\right\rangle=p^{v_{p}\left(\alpha_{j}\right)} \omega\left(g_{j}\right),
$$

and by Corollary 2.5, we have

$$
\left(\begin{array}{c}
\alpha \\
\beta^{(j)}
\end{array}\right) \not \equiv 0 \bmod p
$$

Now suppose that $w\left(\mathbf{g}^{\alpha}-1\right)>t$. We perform binomial expansion in $k G$ to see that

$$
\begin{aligned}
\mathbf{g}^{\alpha}-1 & =\prod_{1 \leq i \leq d}\left(1+b_{i}\right)^{\alpha_{i}}-1 \quad \text { (ordered product) } \\
& =\sum_{\beta \in \mathbb{N}^{d}} \iota\left(\begin{array}{l}
\alpha \\
\beta
\end{array}\right) \mathbf{b}^{\beta}-1 \\
& =\sum_{\beta \neq 0} \iota\left(\begin{array}{l}
\alpha \\
\beta
\end{array}\right) \mathbf{b}^{\beta}
\end{aligned}
$$

so that

$$
w\left(\mathbf{g}^{\alpha}-1\right)=\inf \left\{\langle\beta, \omega(\mathbf{g})\rangle \mid \beta \neq 0,\left(\begin{array}{c}
\alpha \\
\beta
\end{array}\right) \not \equiv 0 \bmod p\right\} .
$$

So in particular, for all $j$ satisfying $\omega\left(g_{j}\right)=t$, we have

$$
t<w\left(\mathbf{g}^{\alpha}-1\right) \leq\left\langle\beta^{(j)}, \omega(\mathbf{g})\right\rangle=p^{v_{p}\left(\alpha_{j}\right)} \omega\left(g_{j}\right),
$$

which is what we wanted to prove.

\subsection{Constructing a suitable valuation}

Let $H$ be a nilpotent $p$-valuable group with centre $Z$. If $k$ is a field of characteristic $p$, and $\mathfrak{p}$ is a faithful prime ideal of $k Z$, then by [2, Theorem 8.4], the ideal $P:=\mathfrak{p} k H$ is again a faithful prime ideal of $k H$.

We will fix the following notation for this subsection.

Notation. Let $G$ be a nilpotent-by-finite compact $p$-adic analytic group, with $\Delta^{+}=1$, and let $H=\mathbf{F N}_{p}(G)$ [10, Definition 5.3], here a nilpotent $p$-valuable radical, so that $\Delta=Z:=Z(H)$ [10, proof of Lemma 1.2.3(iii)]. We will also write $F=G / H$. 
Define $Q^{\prime}=\mathbf{Q}(k Z / \mathfrak{p})$, the (classical) field of fractions of the (commutative) domain $k Z / \mathfrak{p}$, and $Q=Q^{\prime} \underset{k Z}{\otimes} k H$, a tensor product of $k Z$-algebras, which (as $P=\mathfrak{p} k H$ ) we may naturally identify with the (right) localisation of $k H / P$ with respect to $(k Z / \mathfrak{p}) \backslash\{0\}$ - a subring of the Goldie ring of quotients $\mathbf{Q}(k H / P)$.

Suppose further that the prime ideal $\mathfrak{p} \triangleleft k Z$ is invariant under conjugation by elements of $G$.

Choose a crossed product decomposition

$$
k G / P k G=k H / P \underset{\langle\sigma, \tau\rangle}{*} F
$$

which is standard in the sense of the notation of Corollary 1.21. Choose also any $\alpha \in Z_{\sigma}^{2}\left(F, Z\left((k H / P)^{\times}\right)\right)$, and form as in [11, Definition 5.4] the central 2-cocycle twist

$$
(k G / P k G)_{\alpha}=k H / P \underset{\langle\sigma, \tau \alpha\rangle}{*} F .
$$

Now the (right) divisor set $(k Z / \mathfrak{p}) \backslash\{0\}$ is $G$-stable by assumption, so by [8, Lemma 37.7], we may define the partial quotient ring

$$
R:=Q \underset{\langle\sigma, \tau \alpha\rangle}{*} F
$$

Our aim in this subsection is to construct an appropriate filtration $f$ on the ring $R$. We will build this up in stages, following [2]. First, we define a finite set of valuations on $Q^{\prime}$.

Definition 2.7. In [2, Theorem 7.3], Ardakov defines a valuation on $\mathbf{Q}(k H / P)$; let $v_{1}$ be the restriction of this valuation to $Q^{\prime}$, so that $v_{1}(x+\mathfrak{p}) \geq w(x)$ for all $x \in k Z$ (where $w$ is as in Definition 2.3).

Lemma 2.8. $\sigma$ induces a group action of $F$ on the set of valuations of $Q^{\prime}$.

Proof. Let $u$ be a valuation of $Q^{\prime}$. $G$ acts on the set of valuations of $Q^{\prime}$ as follows:

$$
(g \cdot u)(x)=u\left(g^{-1} x g\right) .
$$

Clearly, if $g \in H$, then $g^{-1} x g=x$ (as $x \in \mathbf{Q}(k Z / \mathfrak{p})$ where $Z$ is the centre of $H)$. Hence $H$ lies in the kernel of this action, and we get an action of $F$ on the set of valuations. By our choice of $\bar{F}$ as a subset of the image of $G$, this is the same as $\sigma$.

Write $\left\{v_{1}, \ldots, v_{s}\right\}$ for the $F$-orbit of $v_{1}$.

Lemma 2.9. The valuations $v_{1}, \ldots, v_{s}$ are independent.

Proof. The $v_{i}$ are all non-trivial valuations with value groups equal to subgroups of $\mathbb{R}$ by definition. Hence, by [3, VI.4, Proposition 7], they have height 1. 
They are also pairwise inequivalent: indeed, suppose $v_{i}$ is equivalent to $g \cdot v_{i}$ for some $g \in F$. Then by [3, VI.3, Proposition 3], there exists a positive real number $\lambda$ with $v_{i}=\lambda\left(g \cdot v_{i}\right)$, and so $v_{i}=\lambda^{n}\left(g^{n} \cdot v_{i}\right)$ (as the actions of $\lambda$ and $g$ commute) for all $n$. But $F$ is a finite group: so, taking $n=o(g)$, we get $v_{i}=\lambda^{n} v_{i}$. As $v_{i}$ is non-trivial, we must have that $\lambda^{n}=1$, and so $\lambda=1$. So we may conclude, from [3, VI.4, Proposition 6(c)], that the valuations $v_{1}, \ldots, v_{s}$ are independent.

Definition 2.10. Let $v$ be the filtration of $Q^{\prime}$ defined by

$$
v(x)=\inf _{1 \leq i \leq s} v_{i}(x)
$$

for each $x \in Q^{\prime}$.

Lemma 2.11. $\operatorname{gr}_{v} Q^{\prime} \cong \bigoplus_{i=1}^{s} \operatorname{gr}_{v_{i}} Q^{\prime}$

Proof. The natural map

$$
Q_{v, \lambda}^{\prime} \rightarrow \bigoplus_{i=1}^{s} Q_{v_{i}, \lambda}^{\prime} / Q_{v_{i}, \lambda}^{\prime}
$$

clearly has kernel $\bigcap_{i=1}^{s} Q_{v_{i}, \lambda^{+}}^{\prime}=Q_{v, \lambda^{+}}^{\prime}$, giving an injective map $\operatorname{gr}_{v} Q^{\prime} \rightarrow \bigoplus_{i=1}^{s} \operatorname{gr}_{v_{i}} Q^{\prime}$. The surjectivity of this map now follows from the Approximation Theorem 3 , VI.7.2, Théorème 1], as the $v_{i}$ are independent by Lemma 2.9.

Next, we will extend the $v_{i}$ and $v$ from $Q^{\prime}$ to $Q$, as in the proof of [2, 8.6].

Notation. Continue with the notation above. Now, $H$ is $p$-valuable, and by Lemma 1.20, $F$ acts on the set of $p$-valuations of $H$; hence, by Lemma 1.18 (or Corollary [1.21), we may choose a $p$-valuation $\omega$ which is $F$-stable. Fix such an $\omega$, and construct the valuation $w$ on $k H$ from it as defined in Definition 2.3 .

Let $\left\{y_{e+1}, \ldots, y_{d}\right\}$ be an ordered basis for $Z$, and extend it to an ordered basis $\left\{y_{1}, \ldots, y_{d}\right\}$ for $H$ as in Lemma 1.7 (noting that $Z$ is a closed isolated normal subgroup of $H$ by [2, Lemma 8.4(a)]). For each $1 \leq j \leq e$, set $c_{j}=y_{j}-1$ inside the ring $k H / P$.

Recall from [2, 8.5] that elements of $Q$ may be written uniquely as

$$
\sum_{\gamma \in \mathbb{N} e} r_{\gamma} \mathbf{c}^{\gamma}
$$

where $r_{\gamma} \in Q^{\prime}$ and $\mathbf{c}^{\gamma}:=c_{1}^{\gamma_{1}} \ldots c_{e}^{\gamma_{e}}$, so that $Q \subseteq Q^{\prime}\left[\left[c_{1}, \ldots, c_{e}\right]\right]$ as a left $Q^{\prime}$ module. 
Definition 2.12. For each $1 \leq i \leq s$, as in [2, proof of Theorem 8.6], we will define the valuation $f_{i}: Q \rightarrow \mathbb{R} \cup\{\infty\}$ by

$$
f_{i}\left(\sum_{\gamma \in \mathbb{N}^{e}} r_{\gamma} \mathbf{c}^{\gamma}\right)=\inf _{\gamma \in \mathbb{N}^{e}}\left\{v_{i}\left(r_{\gamma}\right)+w\left(\mathbf{c}^{\gamma}\right)\right\} .
$$

(We remark here a slight abuse of notation: the domain of $w$ is $k H$, and so $w\left(\mathbf{c}^{\gamma}\right)$ must be understood to mean $w\left(\mathbf{b}^{\gamma}\right)$, where $b_{j}=y_{j}-1$ inside the ring $k H$ for each $1 \leq j \leq e$. That is, $b_{j}$ is the "obvious" lift of $c_{j}$ from $k H / P$ to $k H$. This relies on the assumption that $P$ is faithful.)

Note in particular that $\left.f_{i}\right|_{Q^{\prime}}=v_{i}$, and $\operatorname{gr}_{f_{i}} Q$ is a commutative domain, again by [2, proof of Theorem 8.6].

Lemma 2.13. $\sigma$ induces a group action of $F$ on the set of valuations of $Q$.

Proof. Let $u$ be a valuation of $Q$. Again, $G$ acts on the set of valuations of $Q$ by $(g \cdot u)(x)=u\left(g^{-1} x g\right)$. Now, any $n \in H$ can be considered as an element of $Q^{\times}$, so that

$$
(n \cdot u)(x)=u\left(n^{-1} x n\right)=u\left(n^{-1}\right)+u(x)+u(n)=u(x) .
$$

In the following lemma, we crucially use the fact that $\omega$ has been chosen to be $F$-stable.

Lemma 2.14. $f_{1}, \ldots, f_{s}$ is the $F$-orbit of $f_{1}$.

Proof. Take some $g \in F$ and some $1 \leq i, j \leq s$ such that $v_{j}=g \cdot v_{i}$. We will first show that, for all $x \in Q$, we have $f_{j}(x) \leq g \cdot f_{i}(x)$. Indeed, as $\left.f_{j}\right|_{Q^{\prime}}=v_{j}=g \cdot v_{i}=\left.g \cdot f_{i}\right|_{Q^{\prime}}$, and both $f_{j}$ and $g \cdot f_{i}$ are valuations, it will suffice to show that $\left(w\left(c_{k}\right)=\right) f_{j}\left(c_{k}\right) \leq g \cdot f_{i}\left(c_{k}\right)$ for each $1 \leq k \leq e$.

Fix some $1 \leq k \leq e$. Write $y_{k}^{g}=z \mathbf{y}^{\alpha}$ for some $\alpha \in \mathbb{Z}_{p}^{e}$ and $z \in Z$, so that

$$
\begin{aligned}
c_{k}^{g}=y_{k}^{g}-1 & =z \mathbf{y}^{\alpha}-1 \\
& =(z-1)+z\left(\prod_{i=1}^{e}\left(1+c_{i}\right)^{\alpha_{i}}-1\right) \quad \text { (ordered product) } \\
& =(z-1)+z\left(\sum_{\beta \neq 0} \iota\left(\begin{array}{l}
\alpha \\
\beta
\end{array}\right) \mathbf{c}^{\beta}\right),
\end{aligned}
$$

and hence

$$
\begin{array}{rlrl}
\left(g \cdot f_{i}\right)\left(c_{k}\right) & =\inf \left\{v_{i}(z-1), w\left(\mathbf{c}^{\beta}\right) \mid \iota\left(\begin{array}{l}
\alpha \\
\beta
\end{array}\right) \neq 0\right\} & \text { by Definition 2.12 } \\
& \geq \inf \left\{w(z-1), w\left(\mathbf{c}^{\beta}\right) \mid \iota\left(\begin{array}{c}
\alpha \\
\beta
\end{array}\right) \neq 0\right\} & \text { by Definition 2.7 } \\
& =w\left(c_{k}^{g}\right),
\end{array}
$$


with this final equality following from [2, Lemma 8.5(b)]. But now, as $\omega$ has been chosen to be $G$-stable, $w$ is also $G$-stable (see the remark in Definition 2.3), so that $w\left(c_{k}^{g}\right)=w\left(c_{k}\right)$.

Now, we have shown that, if $v_{j}=g \cdot v_{i}$ on $Q^{\prime}$, then $f_{j} \leq g \cdot f_{i}$ on $Q$.

Similarly, we have $v_{i}=g^{-1} \cdot v_{j}$ on $Q^{\prime}$, so $f_{i} \leq g^{-1} \cdot f_{j}$ on $Q$. But $f_{i}(x) \leq f_{j}\left(x^{g^{-1}}\right)$ for all $x \in Q$ is equivalent to $f_{i}\left(y^{g}\right) \leq f_{j}(y)$ for all $y \in Q$ (by setting $x=y^{g}$ ). Hence we have $f_{i}=g \cdot f_{j}$ on $Q$, and we are done.

As in Definition 2.10

Definition 2.15. Let $f$ be the filtration of $Q$ defined by

$$
f(x)=\inf _{1 \leq i \leq s} f_{i}(x)
$$

for each $x \in Q$.

We now verify that the relationship between $f$ and $v$ is the same as that between the $f_{i}$ and the $v_{i}$ (Definition 2.12).

Lemma 2.16. Take any $x \in Q$, and write it in standard form as

$$
x=\sum_{\gamma \in \mathbb{N}^{e}} r_{\gamma} \mathbf{c}^{\gamma} .
$$

Then we have

$$
f(x)=\inf _{\gamma \in \mathbb{N}^{e}}\left\{v\left(r_{\gamma}\right)+w\left(\mathbf{c}^{\gamma}\right)\right\} .
$$

Proof. Immediate from Definitions 2.10, 2.12 and 2.15.

Now we can extend Lemma 2.11 to $Q$ :

Lemma 2.17. $\operatorname{gr}_{f} Q \cong \bigoplus_{i=1}^{s} \operatorname{gr}_{f_{i}} Q$.

Proof. As in the proof of Lemma 2.11, we get an injective map

$$
\operatorname{gr}_{f} Q \rightarrow \bigoplus_{i=1}^{s} \operatorname{gr}_{f_{i}} Q
$$

The proof of $[2,8.6]$ gives a map

$$
\left(\operatorname{gr}_{v}(k Z / \mathfrak{p})\right)\left[Y_{1}, \ldots, Y_{e}\right] \rightarrow \operatorname{gr}_{f}(k H / P)
$$

and isomorphisms

$$
\left(\operatorname{gr}_{v_{i}}(k Z / \mathfrak{p})\right)\left[Y_{1}, \ldots, Y_{e}\right] \cong \operatorname{gr}_{f_{i}}(k H / P)
$$


for each $1 \leq i \leq s$, in each case mapping $Y_{j}$ to $\operatorname{gr}\left(c_{j}\right)$ for each $1 \leq j \leq e$.

Now, gr $k H$ is a gr-free [5, §I.4.1, p. 28] gr $k Z$-module with respect to $f$ and each $f_{i}$, and each of these filtrations is discrete on $k H$ by construction (see [2, Corollary 6.2 and proof of Theorem 7.3]), so by [5, I.6.2(3)], $k H$ is a filt-free $k Z$ module with respect to $f$ and each $f_{i}$; and by [5] I.6.14], these maps extend to a $\operatorname{map}\left(\operatorname{gr}_{v} Q^{\prime}\right)\left[Y_{1}, \ldots, Y_{e}\right] \rightarrow \operatorname{gr}_{f} Q$ and isomorphisms $\left(\operatorname{gr}_{v_{i}} Q^{\prime}\right)\left[Y_{1}, \ldots, Y_{e}\right] \cong \operatorname{gr}_{f_{i}} Q$ for each $i$.

Applying Lemma 2.14 to each $1 \leq i \leq s$, we get isomorphisms

$$
\left(\operatorname{gr}_{v_{i}} Q^{\prime}\right)\left[Y_{1}, \ldots, Y_{e}\right] \rightarrow \operatorname{gr}_{f_{i}} Q
$$

which give a commutative diagram

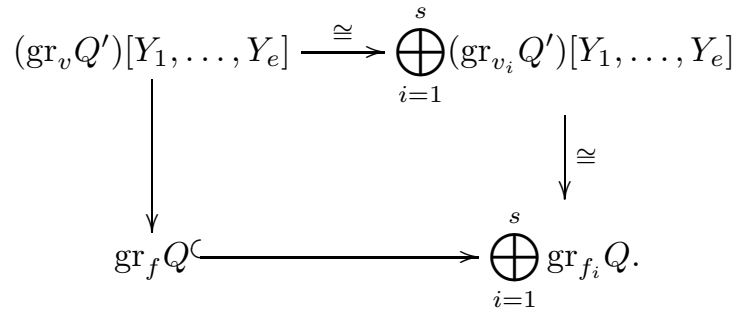

Hence clearly all maps in this diagram are isomorphisms.

Now we return to the ring $R=Q * F$ defined in (3.2).

Definition 2.18. We can extend the filtration $f$ on $Q$ to an $F$-stable filtration on $R$ by giving elements of the basis $\bar{F}$ value 0 . That is, writing $\bar{F}=\left\{\bar{g}_{1}, \ldots, \bar{g}_{m}\right\}$, any element of $Q * F$ can be expressed uniquely as $\sum_{r=1}^{m} \bar{g}_{r} x_{r}$ for some $x_{r} \in Q$ : the assignment

$$
\begin{aligned}
Q * F & \rightarrow \mathbb{R} \cup\{\infty\} \\
\sum_{r=1}^{m} \bar{g}_{r} x_{r} & \mapsto \inf _{1 \leq r \leq m}\left\{f\left(x_{r}\right)\right\}
\end{aligned}
$$

is clearly a filtration on $Q * F$ whose restriction to $Q$ is just $f$. We will temporarily refer to this filtration as $\hat{f}$, though later we will drop the hat and simply call it $f$.

Note that, for any real number $\lambda$,

$$
\begin{aligned}
(Q * F)_{\hat{f}, \lambda} & =\bigoplus_{i=1}^{m} \overline{g_{i}}\left(Q_{f, \lambda}\right), \\
(Q * F)_{\hat{f}, \lambda^{+}} & =\bigoplus_{i=1}^{m} \overline{g_{i}}\left(Q_{f, \lambda^{+}}\right),
\end{aligned}
$$


so that

$$
\begin{aligned}
\operatorname{gr}_{\hat{f}}(Q * F) & =\bigoplus_{\lambda \in \mathbb{R}}\left(\bigoplus_{i=1}^{m} \overline{g_{i}}\left(Q_{f, \lambda} / Q_{f, \lambda^{+}}\right)\right) \\
& =\bigoplus_{i=1}^{m} \overline{g_{i}}\left(\bigoplus_{\lambda \in \mathbb{R}}\left(Q_{f, \lambda} / Q_{f, \lambda^{+}}\right)\right)=\bigoplus_{i=1}^{m} \overline{g_{i}}\left(\operatorname{gr}_{f}(Q)\right)
\end{aligned}
$$

That is, given the data of a crossed product $Q * F$ as in (3.2), we may view $\operatorname{gr}_{\hat{f}}(Q * F)$ as $\operatorname{gr}_{f}(Q) * F$ in a natural way.

We will finally record this as:

Lemma 2.19.

$$
\begin{aligned}
\operatorname{gr}_{f}(Q * F)=\operatorname{gr}_{f}(Q) * F & \cong\left(\bigoplus_{i=1}^{s} \operatorname{gr}_{f_{i}} Q\right) * F \\
& \cong\left(\bigoplus_{i=1}^{s}\left(\operatorname{gr}_{v_{i}} Q^{\prime}\right)\left[Y_{1}, \ldots, Y_{e}\right]\right) * F
\end{aligned}
$$

where each $\operatorname{gr}_{f_{i}} Q$ (or equivalently each $\operatorname{gr}_{v_{i}} Q^{\prime}$ ) is a domain (see Definition 2.12). $F$ permutes the $f_{i}$ (or equivalently the $v_{i}$ ) transitively by conjugation.

Proof of Theorem C. This is Lemma 2.19.

\subsection{Automorphisms trivial on a free abelian quotient}

We will fix the following notation for this subsection.

Notation. Let $H$ be a nilpotent but non-abelian $p$-valuable group with centre $Z$. Write $H^{\prime}$ for its isolated derived subgroup [10, Theorem B]. Suppose we are given a closed isolated proper characteristic subgroup $L$ of $H$ which contains $H^{\prime}$ and $Z$. (We will show that such an $L$ always exists in Lemma 3.5.) Fix a $p$-valuation $\omega$ on $H$ satisfying $\left(\mathrm{A}_{L}\right)$ (which is possible by Corollary 1.21).

Let $\left\{g_{m+1}, \ldots, g_{n}\right\}$ be an ordered basis for $Z$. Using Lemma 1.7 twice, extend this to an ordered basis $\left\{g_{l+1}, \ldots, g_{n}\right\}$ for $L$, and then extend this to an ordered basis $\left\{g_{1}, \ldots, g_{n}\right\}$ for $H$. Diagrammatically:

$$
B_{H}=\{\underbrace{g_{1}, \ldots, g_{l}}_{B_{H / L}}, \underbrace{g_{l+1}, \ldots, g_{m}}_{B_{L / Z}}, \underbrace{g_{m+1}, \ldots, g_{n}}_{B_{Z}}\},
$$

extending the notation of the remark after Lemma 1.7 in the obvious way. Here, $0<l \leq m<n$, corresponding to the chain of subgroups $1 \lesseqgtr Z \leq L \lesseqgtr H$.

Let $k$ be a field of characteristic $p$. As before, let $\mathfrak{p}$ be a faithful prime ideal of $k Z$, so that $P:=\mathfrak{p} k H$ is a faithful prime ideal of $k H$, and write $b_{j}=g_{j}-1 \in k H / P$ for all $1 \leq j \leq m$. 
In this subsection, we will write:

- for each $\alpha \in \mathbb{N}^{m}, \mathbf{b}^{\alpha}$ means the (ordered) product $b_{1}^{\alpha_{1}} \ldots b_{m}^{\alpha_{m}} \in k H / P$,

- for each $\alpha \in \mathbb{Z}_{p}^{m}, \mathbf{g}^{\alpha}$ means the (ordered) product $g_{1}^{\alpha_{1}} \ldots g_{m}^{\alpha_{m}} \in H$,

- for each $\alpha \in \mathbb{N}^{m},\langle\alpha, \omega(\mathbf{g})\rangle$ means $\sum_{i=1}^{m} \alpha_{i} \omega\left(g_{i}\right)$.

Note the use of $m$ rather than $n$ in each case. This means that every element $x \in H$ may be written uniquely as

$$
x=z \mathbf{g}^{\alpha}
$$

for some $\alpha \in \mathbb{Z}_{p}^{m}$ and $z \in Z$; and every element $y \in k H / P$ may be written uniquely as

$$
y=\sum_{\gamma \in \mathbb{N}^{m}} r_{\gamma} \mathbf{b}^{\gamma}
$$

for some elements $r_{\gamma} \in k Z / \mathfrak{p}$.

Recall the definitions of the filtrations $w$ on $k H$ (Definition 2.3), $v$ on $k Z / \mathfrak{p}$ (Definition 2.10) and $f$ on $k H / P$ (Definition 2.15). We will continue to abuse notation slightly for $w$, as in Definition 2.12 ,

Recall also that, as we have chosen $\omega$ to satisfy $\left(A_{L}\right)$, we have that

$$
w\left(b_{1}\right)=\cdots=w\left(b_{l}\right)<w\left(b_{r}\right)
$$

for all $r>l$.

Let $\sigma$ be an automorphism of $H$, and suppose that, when naturally extended to an automorphism of $k H$, it satisfies $\sigma(P)=P$. Hence we will consider $\sigma$ as an automorphism of $k H / P$, preserving the subgroup $H \subseteq(k H / P)^{\times}$.

Corollary 2.20. With the above notation, fix $1 \leq i \leq l$. If $f\left(\sigma\left(b_{i}\right)-b_{i}\right)>f\left(b_{i}\right)$, then $w\left(\sigma\left(b_{i}\right)-b_{i}\right)>w\left(b_{i}\right)$.

Proof. Write in standard form

$$
\sigma\left(b_{i}\right)-b_{i}=\sum_{\gamma \in \mathbb{N}^{m}} r_{\gamma} \mathbf{b}^{\gamma},
$$

for some $r_{\gamma} \in k Z$, and suppose that $f\left(\sigma\left(b_{i}\right)-b_{i}\right)>f\left(b_{i}\right)$. That is, by Lemma 2.16 .

$$
v\left(r_{\gamma}\right)+w\left(\mathbf{b}^{\gamma}\right)>w\left(b_{i}\right)
$$

for each fixed $\gamma \in \mathbb{N}^{m}$.

We will show that $w\left(r_{\gamma}\right)+w\left(\mathbf{b}^{\gamma}\right)>w\left(b_{i}\right)$ for each $\gamma$. We deal with two cases.

Case 1: $w\left(\mathbf{b}^{\gamma}\right)>w\left(b_{i}\right)$. Then, as $w$ takes non-negative values on $k H$, we are already done. 
Case 2: $w\left(\mathbf{b}^{\gamma}\right) \leq w\left(b_{i}\right)$. Then, by $\left(\mathrm{A}_{L}\right)$, we have either $w\left(r_{\gamma}\right)>w\left(b_{i}\right)$ or $w\left(r_{\gamma}\right)=0$. In the former case, we are done automatically, so assume we are in the latter case and $w\left(r_{\gamma}\right)=0$. Then, by [2, 6.2], $r_{\gamma}$ must be a unit in $k Z$, and so $f\left(r_{\gamma}\right)=0$ by Lemma 2.2 a contradiction.

Hence $w\left(r_{\gamma}\right)+w\left(\mathbf{b}^{\gamma}\right)>w\left(b_{i}\right)$ for all $\gamma \in \mathbb{N}^{m}$. But, as $w$ is discrete by [2, 6.2], we may now take the infimum over all $\gamma \in \mathbb{N}^{m}$, and the inequality remains strict.

Let $\sigma$ be an automorphism of $H$, and recall that $H / L$ is a free abelian pro$p$ group of rank $l$. Choose a basis $e_{1}, \ldots, e_{l}$ for $\mathbb{Z}_{p}^{l}$; then the map $g_{i} L \mapsto e_{i}$ for $1 \leq i \leq l$ is an isomorphism $j: H / L \rightarrow \mathbb{Z}_{p}^{l}$. As $L$ is characteristic in $H$ by assumption, $\sigma$ induces an automorphism of $H / L$, which gives a matrix $M_{\sigma} \in G L_{l}\left(\mathbb{Z}_{p}\right)$ under this isomorphism.

Write

$$
\bar{\omega}: H / L \rightarrow \mathbb{R} \cup\{\infty\}
$$

for the quotient $p$-valuation on $H / L$ induced by $\omega$, i.e.

$$
\bar{\omega}(x L)=\sup _{\ell \in L}\{\omega(x \ell)\}
$$

- note that this is just the $(t, p)$-filtration (Definition 1.4), as we have chosen $\omega$ to satisfy $\left(A_{L}\right)$. Then write

$$
\Omega: \mathbb{Z}_{p}^{l} \rightarrow \mathbb{R} \cup\{\infty\}
$$

for the map $\Omega=\bar{\omega} \circ j^{-1}$, the $(t, p)$-filtration on $\mathbb{Z}_{p}^{l}$ corresponding to $\bar{\omega}$ under the isomorphism $j$.

Remark. If $x \in \mathbb{Z}_{p}^{l}$ has $\Omega(x) \geq t+1$, then $x \in p \mathbb{Z}_{p}^{l}$, by the definition of the $(t, p)$-filtration.

We will write $\Gamma(1)=1+p G L_{l}\left(\mathbb{Z}_{p}\right)$ for the first congruence subgroup of $G L_{l}\left(\mathbb{Z}_{p}\right)$, the open subgroup of $G L_{l}\left(\mathbb{Z}_{p}\right)$ whose elements are congruent to the identity element modulo $p$.

Corollary 2.21. With the above notation, if $f\left(\sigma\left(b_{i}\right)-b_{i}\right)>f\left(b_{i}\right)$ for all $1 \leq i \leq l$, then $M_{\sigma} \in \Gamma(1)$.

Proof. We have, for all $1 \leq i \leq l$,

$$
\begin{aligned}
f\left(\sigma\left(b_{i}\right)-b_{i}\right)>f\left(b_{i}\right) & \Longrightarrow w\left(\sigma\left(b_{i}\right)-b_{i}\right)>w\left(b_{i}\right) \quad \text { by Corollary 2.20. } \\
& \Longrightarrow \omega\left(\sigma\left(g_{i}\right) g_{i}^{-1}\right)>\omega\left(g_{i}\right) \quad \text { by Theorem 2.6. }
\end{aligned}
$$

- which is condition (1.1). Now we may invoke Theorem 1.12 .

Corollary 2.22. Suppose now further that $\sigma$ is an automorphism of $H$ of finite order. If $p>2$ and $f\left(\sigma\left(b_{i}\right)-b_{i}\right)>f\left(b_{i}\right)$ for all $1 \leq i \leq l$, then $\sigma$ induces the identity automorphism on $H / L$. 
Proof. We have shown that $M_{\sigma} \in \Gamma(1)$, which is a $p$-valuable (hence torsionfree) group for $p>2$ by [4, Theorem 5.2]; and if $\sigma$ has finite order, then $M_{\sigma}$ must have finite order. So $M_{\sigma}$ is the identity map.

Proof of Theorem D. This now follows from Corollaries 2.21 and 2.22.

Remark. When $p=2, \Gamma(1)$ is no longer $p$-valuable.

Example 2.23. Let $p=2$, and let

$$
H=\overline{\langle x, y, z \mid[x, y]=z,[x, z]=1,[y, z]=1\rangle}
$$

be the (2-valuable) $\mathbb{Z}_{2}$-Heisenberg group. Let $\sigma$ be the automorphism sending $x$ to $x^{-1}, y$ to $y^{-1}$ and $z$ to $z$. Take $L=\overline{\langle z\rangle}$, and $P=0$.

Write $X=x-1 \in k H / P$, and likewise $Y=y-1$ and $Z=z-1$. Now,

$$
\sigma(X)=\sigma(x)-1=x^{-1}-1=(1+X)^{-1}-1=-X+X^{2}-X^{3}+\ldots,
$$

and so $\sigma(X)-X=X^{2}-X^{3}+\ldots($ as char $k=2)$. Hence $f(\sigma(X)-X)=f\left(X^{2}\right)>f(X)$; but

$$
M_{\sigma}=\left(\begin{array}{cc}
-1 & 0 \\
0 & -1
\end{array}\right) \in \Gamma\left(1, G L_{2}\left(\mathbb{Z}_{2}\right)\right),
$$

and in particular $M_{\sigma} \neq 1$.

\section{Extending prime ideals from $\mathbf{F N}_{p}(G)$}

\section{$3.1 \quad \mathrm{X}$-inner automorphisms}

Definition 3.1. We recall the notation of [11, §5]: given $R$ a ring, $G$ a group and a fixed crossed product $S$ of $R$ by $G$, we will sometimes write the structure explicitly as

$$
S=R \underset{\langle\sigma, \tau\rangle}{*} G,
$$

where $\sigma: G \rightarrow \operatorname{Aut}(R)$ is the action and $\tau: G \times G \rightarrow R^{\times}$the twisting.

Furthermore, we say that an automorphism $\varphi \in \operatorname{Aut}(R)$ is $X$-inner if there exist nonzero elements $a, b, c, d \in R$ such that, for all $x \in R$,

$$
a x b=c x^{\varphi} d
$$

where $x^{\varphi}$ denotes the image of $x$ under $\varphi$. Write $\operatorname{Xinn}(R)$ for the subgroup of $\operatorname{Aut}(R)$ consisting of $\mathrm{X}$-inner automorphisms; and, given a crossed product as in the previous paragraph, we will write $\operatorname{Xinn}_{S}(R ; G)=\sigma^{-1}(\sigma(G) \cap \operatorname{Xinn}(R))$.

Lemma 3.2. $R$ a prime ring and $R * G$ a crossed product. Let $G_{\mathrm{inn}}:=\operatorname{Xinn}_{R * G}(R ; G)$.

(i) If $\sigma \in \operatorname{Aut}(R)$ is $\mathrm{X}$-inner, then $\sigma$ is trivial on the centre of $R$. 
(ii) If $H$ is a subgroup of $G$ containing $G_{\text {inn }}$, and $R * H$ is a prime ring, then $R * G$ is a prime ring.

Proof.

(i) This follows from the description of $\mathrm{X}$-inner automorphisms of $R$ as restrictions of inner automorphisms of the Martindale symmetric ring of quotients $\mathrm{Q}_{s}(R)$, and the fact that $Z(R)$ stays central in $\mathrm{Q}_{s}(R)$ : see [8, $\left.\S 12\right]$ for details.

(ii) This follows from [8, Corollary 12.6]: if $I$ is a nonzero ideal of $R * G$, then $I \cap R * G_{\text {inn }}$ is nonzero, and hence $I \cap R * H$ is nonzero.

\section{$3.2 \quad$ Properties of $\mathbf{F N}_{p}(G)$}

We prove here some important facts about the group $\mathbf{F} \mathbf{N}_{p}(G)$ (defined in [10, Theorem C]).

Lemma 3.3. Let $G$ be a nilpotent-by-finite compact $p$-adic analytic group with $\Delta^{+}=1$. Let $H=\mathbf{F N}_{p}(G)$, and write

$$
K:=K_{G}(H)=\left\{x \in G \mid[H, x] \leq H^{\prime}\right\},
$$

where $H^{\prime}$ denotes the isolated derived subgroup of $H$ [10, Theorem B]. Then $K=H$.

Proof. Firstly, note that $K$ clearly contains $H$, by definition of $H^{\prime}$.

Secondly, suppose that $H$ is $p$-saturable. By the same argument as in [10, Lemma 4.3], $K$ acts nilpotently on $H$, and so $K$ acts nilpotently on the Lie algebra $\mathfrak{h}$ associated to $H$ under Lazard's isomorphism of categories [6]. That is, we get a group representation $\operatorname{Ad}: K \rightarrow \operatorname{Aut}(\mathfrak{h})$, and $(\operatorname{Ad}(k)-1)\left(\mathfrak{h}_{i}\right) \subseteq \mathfrak{h}_{i+1}$ for each $k \in K$ and each $i$. (Here, $\mathfrak{h}_{i}$ denotes the $i$ th term in the lower central series for $\mathfrak{h}$.)

Choosing a basis for $\mathfrak{h}$ adapted to the flag

$$
\mathfrak{h} \supsetneq \mathfrak{h}_{2} \supsetneq \cdots \supsetneq \mathfrak{h}_{r}=0,
$$

we see that $\mathrm{Ad}$ is a representation of $K$ for which $\operatorname{Ad}(k)-1$ is strictly upper triangular for each $k \in K$; in other words, Ad :K $\rightarrow \mathcal{U}$, where $\mathcal{U}$ is a closed subgroup of some $G L_{n}\left(\mathbb{Z}_{p}\right)$ consisting of unipotent upper-triangular matrices. Hence the image $\operatorname{Ad}(K)$ is nilpotent and torsion-free.

Furthermore, ker Ad is the subgroup of $K$ consisting of those elements $k$ which centralise $\mathfrak{h}$, and therefore centralise $H$. This clearly contains $Z(H)$. On the other hand, if $k$ centralises $H$, then $k$ is centralised by $H$, an open subgroup of $G$, and so $k$ must be contained in $\Delta$. But $\Delta=Z(H)$ by [10, Lemma 5.1(ii)] 
Hence $K$ is a central extension of two nilpotent, torsion-free compact $p$-adic analytic groups of finite rank, and so is such a group itself; hence $K$ is nilpotent $p$-valuable by [10, Lemma 2.3], and so must be contained in $H$ by definition of $\mathbf{F N}_{p}(G)$.

Now suppose $H$ is not $p$-saturable, and fix a $p$-valuation on $H$. Conjugation by $k \in K$ induces the trivial automorphism on $H / H^{\prime}$, so by [6] it does also on Sat $\left(H / H^{\prime}\right)$, which is naturally isomorphic to Sat $H /(\operatorname{Sat} H)^{\prime}$ by [10, Lemma 3.2]. This shows that $K \subseteq K_{G}(\operatorname{Sat} H)$. But now, writing $\mathfrak{h}$ for the Lie algebra associated to Sat $H$, the same argument as above, mutatis mutandis, shows that $K_{G}($ Sat $H)=H$.

Some properties.

Lemma 3.4. Let $G$ be a compact $p$-adic analytic group with $\Delta^{+}=1$, and write $H=\mathbf{F N}_{p}(G)$. If $H$ is not abelian, then $H / Z=\mathbf{F N}_{p}(G / Z)$.

Proof. $H / Z$ is a nilpotent $p$-valuable open normal subgroup of $G / Z$, so must be contained within $\mathbf{F N}_{p}(G / Z)$. Conversely, the preimage in $G$ of $\mathbf{F N}_{p}(G / Z)$ is a central extension of $Z$ by $\mathbf{F N}_{p}(G / Z)$, two nilpotent and torsion-free groups, and hence is nilpotent and torsion-free, so must be $p$-valuable by [10, Lemma 2.3], which shows that it must be contained within $H$.

Recall that, if $J$ is a closed isolated subgroup of $H$, then there exists a unique smallest isolated orbital subgroup of $H$ containing $J$, which we call its isolator, and denote $\mathrm{i}_{H}(J)$, as in [10, Definition 1.6].

The (closed, isolated orbital, characteristic) subgroup $\mathrm{i}_{H}\left(H^{\prime} Z\right)$ of $H=\mathbf{F N}_{p}(G)$ will be crucial throughout this section, so we record some results.

Lemma 3.5. Let $H$ be a nilpotent $p$-valuable group. If $H$ is not abelian, then $H \neq \mathrm{i}_{H}\left(H^{\prime} Z\right)$.

Proof. Suppose first that $H$ is $p$-saturated, and write $\mathfrak{h}$ and $\mathfrak{z}$ for the Lie algebras of $H$ and $Z$ respectively under Lazard's correspondence [6]. If $\mathfrak{h}=\mathfrak{h}_{2} \mathfrak{z}$ (writing $\mathfrak{h}_{2}$ for the second term in the lower central series of $\mathfrak{h}$ ), then by applying $[\mathfrak{h},-]$ to both sides, we see that $\mathfrak{h}_{2}=\mathfrak{h}_{3}$. But as $\mathfrak{h}$ is nilpotent, this implies that $\mathfrak{h}_{2}=0$, so that $\mathfrak{h}$ is abelian, a contradiction.

When $H$ is not $p$-saturated: note that $\mathrm{i}_{H}\left(H^{\prime} Z\right)=\operatorname{Sat}\left(H^{\prime} Z\right) \cap H$, by [10, Lemma 3.1], and so that $\operatorname{Sat}\left(H / \mathrm{i}_{H}\left(H^{\prime} Z\right)\right) \cong \operatorname{Sat}(H) / \operatorname{Sat}\left(H^{\prime} Z\right)$ by Lemma [10, Lemma 3.2]. Hence $H / \mathrm{i}_{H}\left(H^{\prime} Z\right)$ has the same (in particular non-zero) rank as $\operatorname{Sat}(H) / \operatorname{Sat}\left(H^{\prime} Z\right)$.

Lemma 3.6. Let $G$ be a nilpotent-by-finite compact $p$-adic analytic group with $\Delta^{+}=1$. Let $H=\mathbf{F N}_{p}(G)$, and assume that $H$ is not abelian. Write

$$
M:=M_{G}(H)=\left\{x \in G \mid[H, x] \leq \mathrm{i}_{H}\left(H^{\prime} Z\right)\right\},
$$


where $H^{\prime}$ denotes the isolated derived subgroup of $H$, and $Z$ the centre of $H$. Then $M=H$.

Proof. Clearly $Z \leq M$. We will calculate $M / Z$.

First, note that $\mathrm{i}_{H}\left(H^{\prime} Z\right) / Z$ is an isolated normal subgroup of $H / Z$, as the quotient is isomorphic to $H / \mathrm{i}_{H}\left(H^{\prime} Z\right)$, which is torsion-free. Also, as $\mathrm{i}_{H}\left(H^{\prime} Z\right)$ contains $H^{\prime} Z$ and hence $\overline{[H, H]} Z$ as an open subgroup, clearly $\mathrm{i}_{H}\left(H^{\prime} Z\right) / Z$ contains $\overline{[H, H]} Z / Z$ as an open subgroup, so that $\mathrm{i}_{H}\left(H^{\prime} Z\right) / Z \leq \mathrm{i}_{H / Z}(\overline{[H, H]} Z / Z)$.

Now, $[H / Z, H / Z]=[H, H] Z / Z$ as abstract groups, so by taking their closures followed by their $(H / Z)$-isolators, we see that

$$
(H / Z)^{\prime}=\mathrm{i}_{H / Z}(\overline{[H, H] Z / Z})=\mathrm{i}_{H / Z}(\overline{[H, H]} Z / Z),
$$

so that

$$
\mathrm{i}_{H}\left(H^{\prime} Z\right) / Z=(H / Z)^{\prime} .
$$

But $x \in M$ if and only if $[H, x] \leq \mathrm{i}_{H}\left(H^{\prime} Z\right)$, which is equivalent to $[H / Z, x Z] \leq(H / Z)^{\prime}$, or in other words $x Z \in K_{G / Z}(H / Z)=H / Z$ by Lemma 3.3. So $M / Z=H / Z$, and hence $M=H$.

\subsection{The extension theorem}

Proposition 3.7. Fix a prime $p>2$ and a finite field $k$ of characteristic $p$. Let $G$ be a nilpotent-by-finite compact $p$-adic analytic group with $\Delta^{+}=1$. Suppose $H=\mathbf{F N}_{p}(G)$, and write $F=G / H$. Let $P$ be a $G$-stable, faithful prime ideal of $k H$. Let $(k G)_{\alpha}$ be a central 2-cocycle twist of $k G$ with respect to a standard (Definition 1.19) decomposition

$$
k G=k H \underset{\langle\sigma, \tau\rangle}{*} F,
$$

for some $\alpha \in Z_{\sigma}^{2}\left(F, Z\left((k H)^{\times}\right)\right)$, as in [11, Theorem 5.12]. Then $P(k G)_{\alpha}$ is a prime ideal of $(k G)_{\alpha}$.

Proof. First, we note that the claim that $P(k G)_{\alpha}$ is a prime ideal of $(k G)_{\alpha}$ is equivalent to the claim that

$$
(k G)_{\alpha} / P(k G)_{\alpha}=k H / P \underset{\langle\sigma, \tau \alpha\rangle}{*} F
$$

is a prime ring.

Case 1. Suppose that $G$ centralises $Z$.

If $H$ is abelian, so that $H=Z$, then every $g \in G$ is centralised by $Z$, an open subgroup of $G$. Hence $g \in \Delta$, i.e. $G=\Delta$. But, by [10, Theorem D], $\Delta \leq H$, and so we have $G=H$ and there is nothing to prove. 
So suppose henceforth that $Z \leq H$, and write $L:=\mathrm{i}_{H}\left(H^{\prime} Z\right)$, so that, by Lemma 3.5. we have $L \lesseqgtr H$. As the decomposition of $k G$ is standard, we may view $F$ as a subset of $G$.

The idea behind the proof is as follows. We will construct a crossed product $R * F^{\prime}$, where $R$ is a certain commutative domain and $F^{\prime}$ is a certain subgroup of $F$, with the following property: if $R * F^{\prime}$ is a prime ring, then $P(k G)_{\alpha}$ is a prime ideal. Then, by using the well-understood structure of $R$, we will show that the action of $F^{\prime}$ on $R$ is $\mathrm{X}$-outer (in the sense of Definition 3.1), so that $R * F^{\prime}$ is a prime ring.

By Corollary 1.21 , we can see that $H$ admits an $F$-stable $p$-valuation $\omega$ satisfying $\left(\mathrm{A}_{L}\right)$. Hence, in the notation of 2.1, we may define the filtration $w$ from $\omega$ as in Definition 2.3. Furthermore, we write

$$
Q^{\prime}=\mathbf{Q}(k Z / P \cap k Z), \quad Q=Q_{k Z}^{\prime} \underset{k Z}{\otimes} k N,
$$

as in 22.2 and we endow $Q$ with the $F$-orbit of filtrations $f_{i}(1 \leq i \leq s)$ and the filtration $f$ of Definitions 2.12 and 2.15, defined in terms of the filtration $w$ above.

By [7, 2.1.16(vii)], in order to show that the crossed product

$$
k H / P \underset{\langle\sigma, \tau \alpha\rangle}{*} F
$$

is a prime ring, it suffices to show that the related crossed product

$$
Q \underset{\langle\sigma, \tau \alpha\rangle}{*} F
$$

is prime, where this crossed product is defined in 2.2 Then, by [5, II.3.2.7], it suffices to show that

$$
\operatorname{gr}_{f}(Q * F)
$$

is prime. Details of this graded ring are given in Lemma 2.19, in particular, note that

$$
\operatorname{gr}_{f}(Q * F) \cong\left(\bigoplus_{i=1}^{s} \operatorname{gr}_{f_{i}} Q\right) * F .
$$

Now, as noted in Definition 2.12, each $\operatorname{gr}_{f_{i}} Q$ is a commutative domain, and by construction, $F$ permutes the summands $\operatorname{gr}_{f_{i}} Q$ transitively. So by $[\underline{8}$, Corollary 14.8] it suffices to show that

$$
\operatorname{gr}_{f_{1}} Q * F^{\prime}
$$

is prime, where $F^{\prime}=\operatorname{Stab}_{F}\left(f_{1}\right)$. 
Notation. We set up notation in order to be able to apply the results of $\$ 2.3$. Let $\left\{y_{m+1}, \ldots, y_{n}\right\}$ be an ordered basis for $Z$, which we extend to an ordered basis $\left\{y_{l+1}, \ldots, y_{n}\right\}$ for $L$, which we extend to an ordered basis $\left\{y_{1}, \ldots, y_{n}\right\}$ for $H$. Set $b_{i}=y_{i}-1 \in k H / P$, and let $Y_{i}=\operatorname{gr}_{f_{1}}\left(b_{i}\right)$ for all $1 \leq i \leq m$. Then

$$
\operatorname{gr}_{f_{1}} Q \cong\left(\operatorname{gr}_{v_{1}} Q^{\prime}\right)\left[Y_{1}, \ldots, Y_{m}\right]
$$

The ring on the right-hand side inherits a crossed product structure

$$
\left(\operatorname{gr}_{v_{1}} Q^{\prime}\right)\left[Y_{1}, \ldots, Y_{m}\right] * F^{\prime} .
$$

from (3.4). Writing $R:=\left(\operatorname{gr}_{v_{1}} Q^{\prime}\right)\left[Y_{1}, \ldots, Y_{m}\right]$, we have now shown, by passing along the chain

$$
\text { (3.5) } \rightarrow \text { (3.4) } \rightarrow(3.3) \rightarrow(3.2) \rightarrow(3.1)
$$

that we need only show that $R * F^{\prime}$ is prime.

Write $F_{\text {inn }}^{\prime}$ for the subgroup of $F^{\prime}$ acting on $R$ by X-inner automorphisms in the crossed product (3.5), i.e.

$$
F_{\text {inn }}^{\prime}=\operatorname{Xinn}_{R * F^{\prime}}\left(R ; F^{\prime}\right)
$$

in the notation of Definition 3.1. By the obvious abuse of notation, we will denote this action as the map of sets gr $\sigma: F^{\prime} \rightarrow \operatorname{Aut}(R)$.

Take some $g \in F^{\prime}$. If gr $\sigma(g)$ acts non-trivially on $R$, then as $R$ is commutative, we have $g \notin F_{\mathrm{inn}}^{\prime}$. Hence, as by Lemma 3.2 (ii) we need only show that $R * F_{\mathrm{inn}}^{\prime}$ is prime, we may restrict our attention to those $g \in F^{\prime}$ that act trivially on $R$. In particular, such a $g \in F^{\prime}$ must centralise each $Y_{i}$. But

$$
\operatorname{gr} \sigma(g)\left(Y_{i}\right)=Y_{i} \Leftrightarrow f\left(\sigma(g)\left(b_{i}\right)-b_{i}\right)>f\left(b_{i}\right) .
$$

Now we see (as $p>2$ ) from Corollary 2.22 that $\sigma(g)$ induces the identity automorphism on $H / L$, and hence from Lemma 3.6 that $g \in H$. That is, $F_{\text {inn }}^{\prime}$ is the trivial group, so that $R * F_{\mathrm{inn}}^{\prime}=R$ is automatically prime.

Case 2. Suppose some $x \in F$ does not centralise $Z$. Write $F_{\text {inn }}$ for the subgroup of $F$ acting by $\mathrm{X}$-inner automorphisms on $k H / P$ in the crossed product (3.1), i.e.

$$
F_{\text {inn }}:=\operatorname{Xinn}_{(k G)_{\alpha} / P(k G)_{\alpha}}(k H / P ; F) .
$$

Then, by Lemma 3.2(i), $x \notin F_{\text {inn }}$; so $F_{\text {inn }}$ is contained in $\mathbf{C}_{F}(Z)$, and we need only prove that the sub-crossed product $(k H / P) * \mathbf{C}_{F}(Z)$ is prime by Lemma 3.2(ii). This reduces the problem to Case 1.

Proposition 3.8. Let $G$ be a nilpotent-by-finite compact $p$-adic analytic group, and $k$ a finite field of characteristic $p>2$. Let $H=\mathbf{F N}_{p}(G)$, and write $F=G / H$. Let $P$ be a $G$-stable, almost faithful prime ideal of $k H$. Then $P k G$ is prime. 
Proof. We assume familiarity with [11, Lemma 1.1], and adopt the notation of [11, Notation 1.2] for this proof.

Let $e \in \mathrm{cpi}^{\overline{k \Delta^{+}}}(P)$, and write $f_{H}=\left.e\right|^{H}, f=\left.e\right|^{G}$. Then $P k G$ is a prime ideal of $k G$ if and only if $f \cdot \overline{P k G}$ is prime in $f \cdot \overline{k G}$.

Write $H_{1}=\operatorname{Stab}_{H}(e)$ and $G_{1}=\operatorname{Stab}_{G}(e)$. Then, by the Matrix Units Lemma [11, Lemma 6.1], we get an isomorphism

$$
f \cdot \overline{k G} \cong M_{s}\left(e \cdot \overline{k G_{1}}\right)
$$

for some $s$, under which the ideal $f \cdot \overline{P k G}$ is mapped to $M_{s}\left(e \cdot \overline{P_{1} k G_{1}}\right)$, where $P_{1}$ is the preimage in $k H_{1}$ of $e \cdot \bar{P} \cdot e$. It is easy to see that $P_{1}$ is prime in $k H_{1}$; indeed, applying the Matrix Units Lemma to $k H$, we get

$$
f_{H} \cdot \overline{k H} \cong M_{s^{\prime}}\left(e \cdot \overline{k H_{1}}\right),
$$

under which $f_{H} \cdot \bar{P} \mapsto M_{s^{\prime}}\left(e \cdot \overline{P_{1}}\right)$, so that $P_{1}$ is prime by Morita equivalence (see e.g. [11, Lemma 1.7]). We also know from [11, Lemma 6.6] (or the remark after [11, Lemma 6.2]) that

$$
P^{\dagger}=\bigcap_{h \in H}\left(P_{1}^{\dagger}\right)^{h}
$$

Now, writing $q$ to denote the natural map $G \rightarrow G / \Delta^{+}$,

$$
q\left(\left(P_{1}^{\dagger} \cap \Delta\right)^{h}\right)=q\left(P_{1}^{\dagger} \cap \Delta\right)
$$

for all $h \in H$, as $q(\Delta)=Z(q(H))$ by definition of $H$ (see [10, Lemma 5.1(ii)]); and so

$$
q\left(P^{\dagger} \cap \Delta\right)=q\left(P_{1}^{\dagger} \cap \Delta\right)=q(1) .
$$

But $q\left(P_{1}^{\dagger}\right)$ is a normal subgroup of the nilpotent group $q\left(H_{1}\right)$. Hence, as the intersection of $q\left(P_{1}^{\dagger}\right)$ with the centre $q(\Delta)$ of $q(H)$ is trivial, we must have that $q\left(P_{1}^{\dagger}\right)$ is trivial also [9, 5.2.1]. That is, $P_{1}^{\dagger} \leq \Delta^{+}\left(H_{1}\right)=\Delta^{+}$.

Now, in order to show that $M_{s}\left(e \cdot \overline{P_{1} k G_{1}}\right)$ is prime, we may equivalently (by Morita equivalence) show that $e \cdot \overline{P_{1} k G_{1}}$ is prime. By [11, Theorem 5.12], we get an isomorphism

$$
e \cdot \overline{k G_{1}} \cong M_{t}\left(\left(k^{\prime}\left[\left[G_{1} / \Delta^{+}\right]\right]\right)_{\alpha}\right),
$$

for some integer $t$, some finite field extension $k^{\prime} / k$, and a central 2-cocycle twist (see above or [11, Definition 5.4]) of $k^{\prime}\left[\left[G_{1} / \Delta^{+}\right]\right]$with respect to a standard crossed product decomposition

$$
k^{\prime}\left[\left[G_{1} / \Delta^{+}\right]\right]=k^{\prime}\left[\left[H_{1} / \Delta^{+}\right]\right] \underset{\langle\sigma, \tau\rangle}{*}\left(G_{1} / H_{1}\right)
$$


given by some

$$
\alpha \in Z_{\sigma}^{2}\left(G_{1} / H_{1}, Z\left(\left(k^{\prime}\left[\left[H_{1} / \Delta^{+}\right]\right]\right)^{\times}\right)\right) .
$$

Writing the image of $e \cdot \overline{P_{1}}$ as $M_{t}(\mathfrak{p})$ for some ideal $\mathfrak{p} \in k^{\prime}\left[\left[H_{1} / \Delta^{+}\right]\right]$, we see by (see above or [11, Theorem $\mathrm{C}]$ ) that $\mathfrak{p}$ is a faithful, $\left(G_{1} / \Delta^{+}\right)$-stable prime ideal of $k^{\prime}\left[\left[H_{1} / \Delta^{+}\right]\right]$. It now remains only to show that the extension of $\mathfrak{p}$ to $k^{\prime}\left[\left[G_{1} / \Delta^{+}\right]\right]$is prime; but this now follows from Proposition 3.7 .

Proof of Theorem A. This follows from Proposition 3.8.

\section{References}

[1] R.C. Alperin. p-adic binomial coefficients MOD P. The American Mathematical Monthly, 92(8):576-578, 1985.

[2] K. Ardakov. Prime ideals in nilpotent Iwasawa algebras. Inventiones mathematicae, 190(2):439-503, 2012.

[3] N. Bourbaki. Commutative Algebra. Springer, 1998.

[4] J.D. Dixon, M.P.F. du Sautoy, A. Mann and D. Segal. Analytic Pro-p Groups. Cambridge University Press, 1999.

[5] Li Huishi and Freddy van Oystaeyen. Zariskian Filtrations. Springer Science+Business Media, 1996.

[6] Michel Lazard. Groupes analytiques p-adiques. Publications Mathématiques de l'IHÉS, 26:5-219, 1965.

[7] J.C. McConnell and J.C. Robson. Noncommutative Noetherian Rings. American Mathematical Society, 2001.

[8] Donald S. Passman. Infinite Crossed Products. Academic Press Inc., 1989.

[9] Derek J.S. Robinson. A course in the theory of groups. Springer-Verlag New York, 1996.

[10] W. Woods. On the structure of virtually nilpotent compact $p$-adic analytic groups. Preprint, arXiv:1608.03137 [math.GR], 2016. To appear in $J$. Group Theory.

[11] W. Woods. Maximal prime homomorphic images of mod- $p$ iwasawa algebras. Preprint, arXiv:1608.07755 [math.RT], 2016. 